\title{
A Mathematical Model for Cellular Locomotion Exhibiting Chemotaxis
}

\author{
M.J. HOLMES ${ }^{*}$ and B.D. SLEEMAN ${ }^{\dagger}$ \\ School of mathematics, Department of Applied Mathematics, University of Leeds, Leeds LS2 9JT, UK
}

(Received 3 February 2000; Revised 23 May 2000; In final form 12 June 2000)

\begin{abstract}
A fundamental problem of cellular biology is to understand the mechanisms underlying cellular locomotion. Bacterial organisms may use appendages such as flagellae or cilia to facilitate motion. Amoeboid motion [6], exhibited by eucaryotic cells are seen to flatten onto surfaces and extend thin sheets of cytosol called lamellipodia. These in turn make attachments to the surface and by the initiation of internal contractions within the cell, a forward motion is achieved. The processes which govern this behaviour are extremely complex, however, key ingredients have been identified which may provide a sufficient basis for persistent cellular motion. These factors are osmotic/hydrostatic expansion and cellular contraction mediated by intracellular calcium $\mathrm{Ca}^{2+}$. In this paper, we develop a simple two dimensional model for a non-muscle motile cell based on these two key factors. We show it is capable of producing persistent cellular motion and chemotactic behaviour.
\end{abstract}

\section{INTRODUCTION}

A fundamental problem of cellular biology is to understand the mechanisms underlying cellular locomotion. Bacterial organisms may use appendages such as flagellae or cilia to facilitate motion. Also, bacterium migrate up chemotactic gradients by effectively taking measurements in time. In amoeboid motion, [6], more sophisticated principles are implemented to achieve locomotion and chemotaxis, the main features of which will be described later in the text.

In order for an organism as a whole to develop and maintain a functioning form, it is essential that its constituent cells have the capacity to move. However, not all such cells appear to have motile abilities. Fibrob- lasts, endothelial cells and neutrophils show high motile capabilities, whilst epithelial cells when cultured individually, show little or no motion at all [3]. Each is acting in accordance with either an inherent programme or is being stimulated by some external signal. For example, when neutrophils or macrophages detect bacteria they quickly migrate to the infection site, in response to a chemical signal to ingest the invading organisms. As we shall reveal, the mechanisms which cells utilise in order to move and interact with their external environment are immensely complex.

Both microscopic and macroscopic models have been developed to explain the behaviour of cells. Obviously the internal aspects of cellular locomotion being considered in the former, whilst the latter

\footnotetext{
* E' mail amtmh@amsta.leeds.ac.uk

$\dagger E^{\prime}$ mail bds@amsta.leeds.ac.uk
} 
focuses on the interaction of populations of cells. On the macroscopic scale many continuum models have been developed to simulate for example angiogenesis and morphogenesis [23], [28], [29], [31], [32], [34], [35], [41]. Typically these models might incorporate cellular traction, extracellular matrix properties and chemotactic responses and examine the resulting evolution of cellular densities.

At a cellular level the processes involved in motion are not fully understood due to the complexity of the metabolic and mechanical processes involved. Due to their relatively large size, amoeba have been studied in great detail to understand cellular motion. In amoeba the central core of fluid containing the organelles is called the endoplasm or sol. The outer fluid which is generally transparent and free from organelles is called ectoplasm or gel and can be made very rigid by alteration of the actin network present. Eucaryotic cells, such as fibroblasts and endothelial cells, however, are of the order ten times smaller in length and therefore more difficult to observe, although it is thought the processes involved are similar. Any reference to cells henceforth will relate to motile eucaryotic cells unless otherwise is stated. These types of cells are seen to flatten onto surfaces and extend thin sheets of cytosol called lamellipodia. These in tum make attachments to the surface and by the initiation of internal contractions within the cell, a forward motion is achieved. Also it is observed that the cell membrane is continually flowing to the rear of the cell. When lamellae fail to make attachments to the surface, they are drawn back into the cell body giving a characteristic 'ruffle' like appearance on the cell membrane. In the course of the extension-contraction cycle a series of elaborate mechanisms come into play and several models have been proposed, based on known physical principles, to elucidate the behaviour, see for example [13], [27], [37], [40] and [46]. A characteristic property of cells, which some of these models incorporate, is the visco-elastic nature of the cytoskeleton. This particular view point is one which we shall adopt and we will describe in detail later in the text. In contrast, the models developed in [4] and [5] use fluid dynamical ideas to describe the 'ruffle' effect and the complex membrane variations which are observed, such as invagination.
The main constituents of a cell volume consist of an aqueous solution of water, ions and macromolecular proteins. One such protein which exists in abundance is globular monomeric actin, or G-actin. Actin exists in essentially two forms, G-actin and F-actin. $\mathrm{G}$-actin has a globular monomeric form and upon polymerization G-actin attains a helical filamentous structure which is called F-actin. Typically these filaments are about $5-7 \mathrm{~nm}$ in diameter and $38 \mathrm{~nm}$ in length. Additionally, each actin filament is polarized, the two ends having quite different properties and conventionally described as the barbed and pointed ends. The barbed ends of the filaments usually attach to the plasma membrane at the lamellae and the pointed ends to the main cell body. G-actin must exist at a critical concentration level before polymerization of F-actin can occur, typically the level of G-actin which exists in human leukocytes is three times higher than this critical concentration. The regulation of actin polymerization is controlled by the formation of a complex called profilactin which consists of G-actin and profilin, a polymerization inhibitor [46]. This closely correlates with the $\mathrm{Ca}^{2+}$ calcium concentration present which we will discuss shortly. Actin plays an essential role in cellular locomotion. The interactions of actin with light and heavy chain myosin [3] provide the mechanisms for generating coherent contractile forces. This is achieved by conformational changes in the myosin molecule. A further important ingredient for cellular motion is that of osmotic/hydrostatic pressure. In order to extend a lamellae the cell must generate a propulsive force. However, when cells are placed in a hypertonic solution (higher concentration), all lamellae protrusions ceased [19], [43], thus implicating osmotic pressure as a candidate for the force generation. Due to the isotropic nature of osmotic pressure, a coordinating process must exist to focus the protrusion at a particular point on the membrane. This is accomplished by stimulation of the leading edge membrane receptors by some external chemoattractant. This may initiate a sequence of events which culminates in the release of internal stores of calcium $\mathrm{Ca}^{2+}$ contained in various organelles and allows influx of ions suchs as $\mathrm{Ca}^{2+}$. This in turn activates solation factors within the cyto- 
gel which sever actin filaments and reduce the associated elastic modulus cytogel. As a result of this, the volume fraction of crosslinked cytogel is reduced. A combination of intracellular hydrostatic pressure and osmotic pressure extend the lamellae further where it re-attaches to the extra-cellular matrix (ECM) [6]. Also, the influx of $\mathrm{Ca}^{2+}$ through the plasma membrane will remodel the filaments in the cortical layer, this process itself is mediated by actin fragmenting proteins such as gelsolin and villin [3], [26], [43].

Calcium $\mathrm{Ca}^{2+}$ has been implicated as a basic requirement to produce cellular motion since it initiates actin polymerization and can also stimulate local contraction within a cell at the site of deposition [6]. Additionally, calcium instigates nucleation of new actin fibres and crosslinking proteins which promote reformation of the cytogel. This stabilises the lamellipod by increasing the elastic modulus thus reducing the effect of osmotic extension. Proteins such as fimbrin hold actin fibres in parallel arrays. Others like $\alpha$-actinin and filamin have the capacity to hold fibres in any configuration. Since there is an increase of $\mathrm{Ca}^{2+}$ within the cortical layer during the influx there exists a mechanism to actively remove it. This is accomplished by transport into cellular organelles such as the endoplasmic reticulum which specialises in the transport and synthesis of membrane proteins. When a critical threshold is reached, the transient effect of osmotic extension is inhibited, $\mathrm{Ca}^{2+}$ then additionally triggers phosphorylation of myosin light chain kinase which causes contraction of the actomyosin gel, which in turn increases as the calcium is resequestered into the cell [6]. This is a slower process than expansion and we assume it is has negligible contribution during the expansion phase and only becomes important once the lamellae are stabilised. After chemical proteins have bound to surface receptors they are ingested by receptor-mediated endocytosis. A degradation or down regulation of ligand-receptors is then experienced culminating in a desensitization of the cell membrane.

An axisymmetric distribution of adhesion ligand-receptors on the cell surface at the lamellae and basal region offers the potential for motile behaviour. Provided the contraction of the cytoskeleton is suffi- ciently strong to break the cellular attachments to the surrounding medium, the cell is able to draw its basal region forward. Later in the text we will utilise the fact that substrate adhesion bonds are broken to enable cell motion. As the cell moves forward, often a long fibre is seen to be drawn out at the rear of the cell. This can suddenly be retracted once the adherence to the underlying membrane is overcome. Figure 1 (reproduced from [26]) shows the basic stages of cell motion.

Substantial investigations have been carried out in order to quantify the physical properties of cells such as the cytoplasmic viscosity, elasticity and also the traction which may be generated by a cell. Many other properties have been investigated which address the adhesion receptor-ligand kinetics, see for example [1], [6], [16], [17], [39], [45]. Quantification of these parameters in themselves pose demanding experimental problems.

When addressing the problem of chemotaxis one must also account for the phenomenon of chemokinesis. Chemokinesis is a process whereby a particular agent may affect the rate of locomotion. This may cause a cellular population to cluster in a particular location, giving the impression a population of cells have detected the spatial variation of the agent. Experiments are necessarily more complex then to account for this fact. In contrast, chemotaxis is a process whereby a cell detects spatial variation of an agent across its own dimensions and subsequently changes its direction of locomotion accordingly. Thus, for a cellular model to capture the chemotactic property it must be able to detect the spatial variation of some external agent. The chemotactic stimulation of a cell correlates strongly with the internal calcium ion concentration. Typically the level of concentration of calcium $\mathrm{Ca}^{2+}$ external to the cell is of the order $10^{-3} \mathrm{M}$ or greater, whilst the internal concentration of free $\mathrm{Ca}^{2+}$ in the cytosol is much lower, around $10^{-7} \mathrm{M}$. As such, there is a large resource of $\mathrm{Ca}^{2+}$ for the cell to utilise. Activating receptors on the cell membrane may raise the internal concentration of $\mathrm{Ca}^{2+}$ very quickly by initiating the transient opening of channel pathways. This is of particular importance with muscle cells which require a fast response mechanism. The process is slower for cells such as fibroblasts and epitheliocytes. 

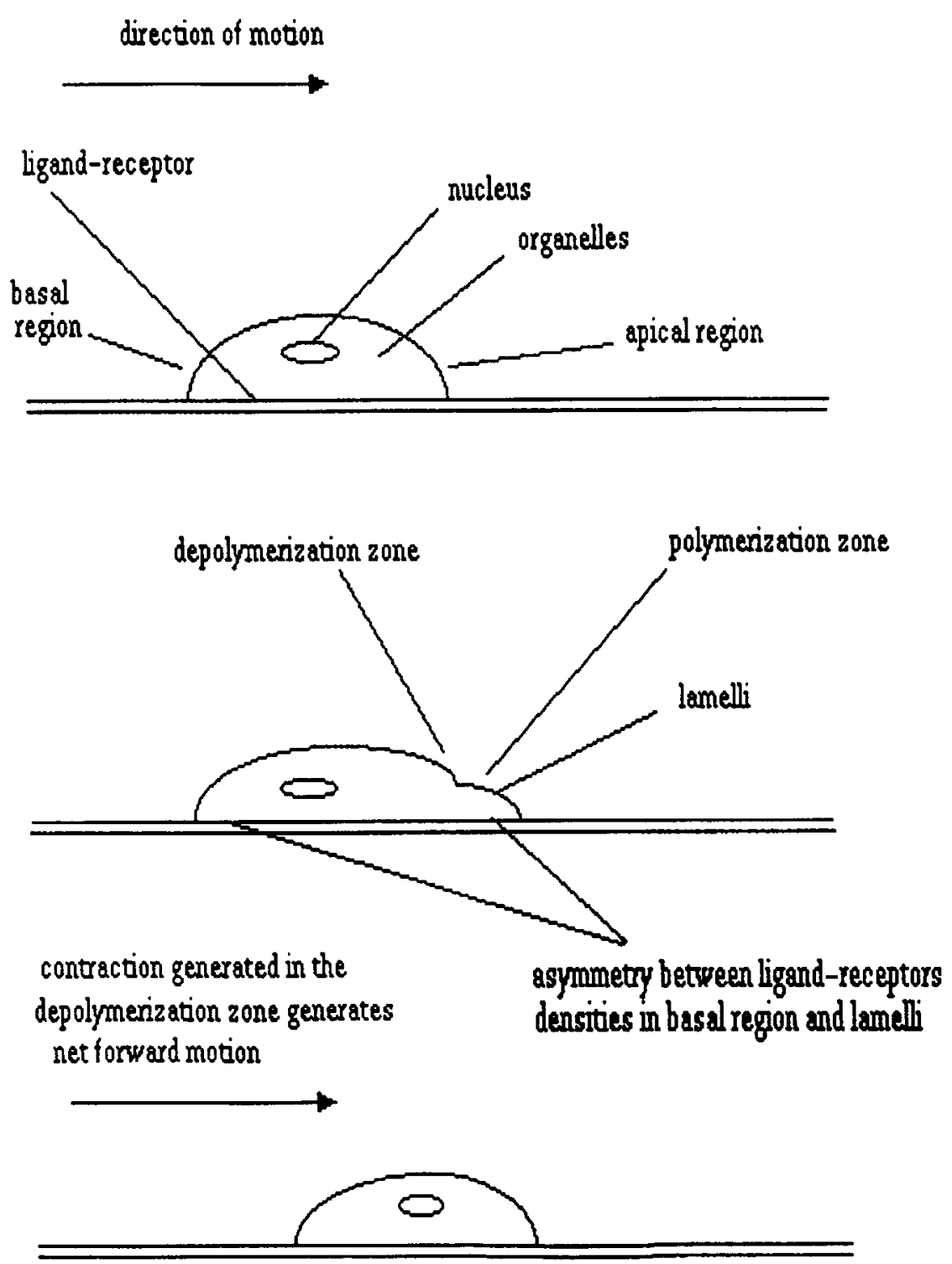

FIGURE 1 Simplified stages involved in cellular locomotion. Redrawn from [26]

The influx of $\mathrm{Ca}^{2+}$ ceases when the intracellular and extracellular concentrations equate, or, the plasma membrane channels are closed. $\mathrm{Ca}^{2+}$ influx as stated above, is also supplemented by the release of internal stores contained in organelles such as the endoplasmic reticulum or in the case of muscle cells the sarcoplasmic reticulum. This accumulation of
$\mathrm{Ca}^{2+}$ will subsequently be resequestered by the same organelles and be consumed in various metabolic pathways. This cycling process of $\mathrm{Ca}^{2+}$ provides a mechanism for myofibril contraction/relaxation. Figure 2 shows the timescales in which the sarcoplasmic reticulum is able to accumulate free $\mathrm{Ca}^{2+}$ from the cytosol and release those stores with the addition 


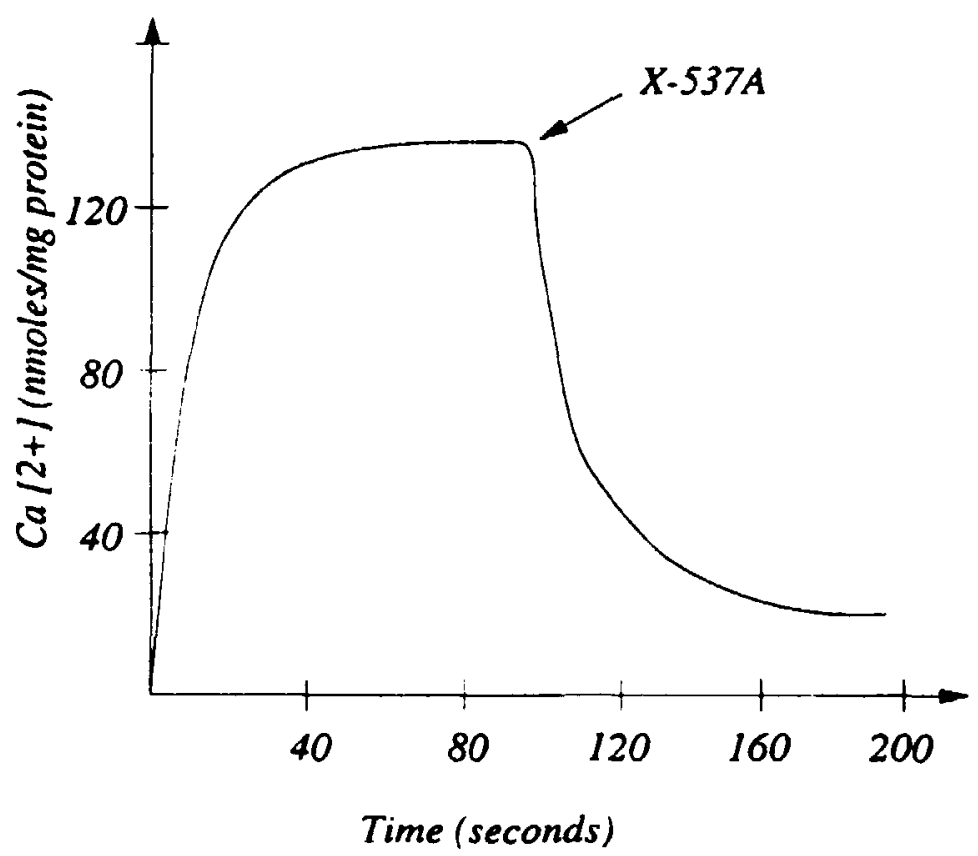

FIGURE 2. Timescales of uptake and $\mathrm{Ca}^{2+}$ release by sarcoplasmic reticulum vesicles. Introduction of the ionophore $\mathrm{X}-537 \mathrm{a}$ instigates the release of stored $\mathrm{Ca}^{2+}$. Taken from [15]

of a suitable agent. Chemotactic receptors also have the ability to alter the cell membrane permeability which in turn affects the ionic influx. In [48], chemotactic influences were observed to change both the influx and efflux of various radioactive elements including calcium. As in [13], it is assumed for each cell cycle the above phases are placed into separate time periods. The extension phase is defined as the time period given by the set $T_{e}=\left(t \in \mathrm{R}: 0 \leq t \leq t_{e}\right)$ and the combined contraction/relaxation period is defined for the set $T=\left(t \in \mathrm{R}: t_{e}<t\right)$. The extension phase $T_{\epsilon}$ correlates to the time period during which the cell receives an influx of $\mathrm{Ca}^{2+}$ and the channel pathways are open. During this phase the osmotic pressure extends the lamellipod. The duration of the time period $T$ corresponds to the contraction/relaxation time, this being dependent upon the chemical kinetics within the cell. The presence of intracellular $\mathrm{Ca}^{2+}$ in the cortical layer initiates the myofibril contraction. This will persist until the calcium concentra- tion is resequestered and restored to the original concentration of about $10^{-7} \mathrm{M}$. Once the internal calcium returns to its initial steady state value, the cell again becomes receptive to further external stimulus. Effectively, at this point $t$ is reset so that $t=0$ and the cycle repeats. It is observed [6] that the lamellipod is significantly thinner than the cell body being approximately $0.1-1.0 \mu m$ thick. Thus we treat the cell as a two-dimensional object where we are examining a thin slice through the length of the cell in the plane through the lamellipod to basal region, refer (Figure 3). We impose an explicit functional form to simulate opening of the channel pathways at the apical region of the cell during the stimulatory phase of the cell motion. Thus we do not address receptor kinetics or the cell's control mechanism for allowing influx of $\mathrm{Ca}^{2+}$ or chemoattractant binding at the lamellipod. We allow influx of $\mathrm{Ca}^{2+}$ for the time period $T_{e}$ and then make the leading edge or lamellae impermeable for the time period $T$. 


\section{plane section taken through cell}

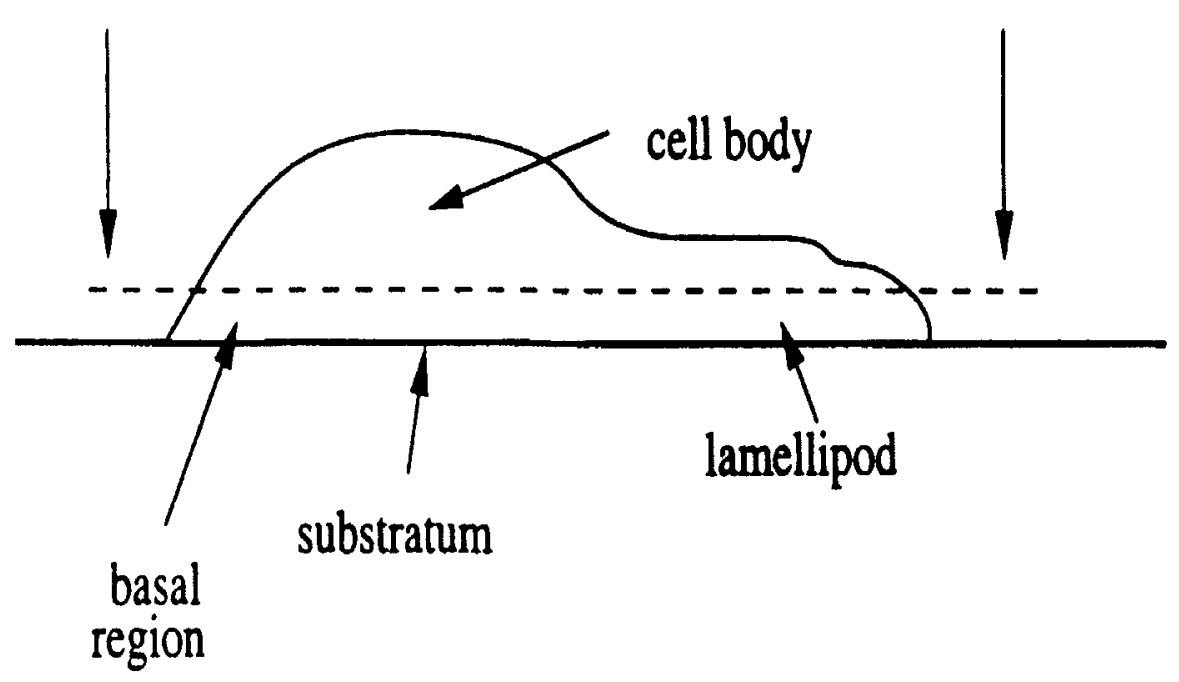

FIGURE 3 Idealised model cell is taken as a plane section through the cell body

Also, we assume that adhesion to the surface is at ideal state, that is, when calcium is released within the cell the attachments to the substratum are severed and no frictional forces are generated. Only cellular attachments at the basal region are maintained during the extension phase and cellular attachments at the lamellipod during the contractile/relaxation phase. Alternatively, one could interpret this as the extensile forces generated within the cell at the points of calcium release having sufficient strength to overcome the resistive forces presented by the substrate. If a net forward translocation is to be achieved, then the substrate adhesion force must be less than the traction force generated by the cell in order to break the bonds to the substrate. However, there is evidence [7] to indicate that cells may be haptotactic. That is, a cell may actively migrate up an adhesive gradient and so future models could address the ligand-receptor interaction of the cell with the extracellular matrix.

In following sections of the paper we develop a two dimensional system to simulate the behaviour of a cell relative to some external chemoattractant. We couple this with the associated calcium influx and subsequent osmotic expansion and actomyosin contractions which enable cellular motion.

The disparity of spatial and temporal scales in connection with cell lengths and migration distances and also internal cellular kinetic timescales, makes the modelling of individual cells in connection with migration over relatively large length scales difficult. It is therefore our intention to consider a simplifed system of a single cell and examine its behaviour in a local environment. That is, we treat the cell as a visco-elastic material and relate intracellular calcium concentration to osmotic pressure and cellular traction. The internal calcium concentration itself is modulated by the influx through the cell membrane, which in turn is governed by the external chemoattractant present. This allows the cell to interact with its local environment by assuming chemotactic incentives influence the cell's behaviour. In particular, this would result in deformations and motile behaviour of the cell toward the local chemotactic source.

The system is broadly based on a model proposed by [36] to address the internal aspects of cellular locomotion. Also, as we shall describe further in the text, 


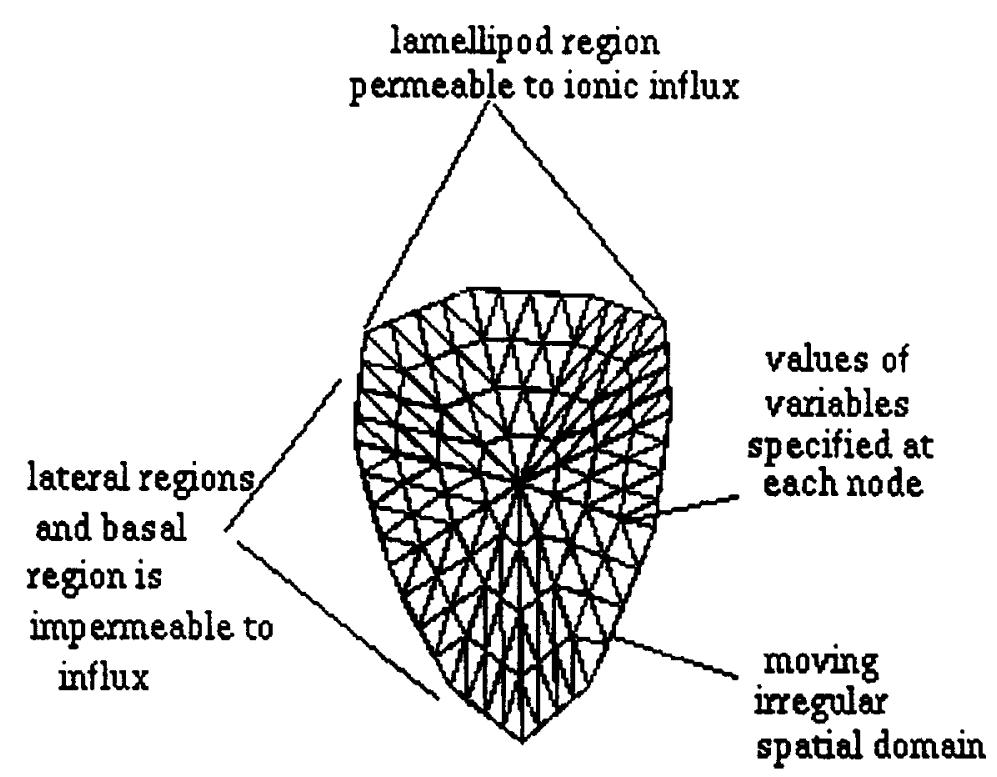

FIGURE 4 Conceptualised view of a cell

we embed the cell in a domain where we may prescribe certain functional forms which may represent external concentration of some chemotactic agent. This could be realised as a tumour secreting angiogenic factors in order to stimulate the migration of endothelial cells. Since the chemoattractant has the ability to influence the plasma membrane permeability, we correlate the external chemoattractant to that of the calcium $\mathrm{Ca}^{2+}$ present at the lamellipod membrane. This then directly influences the cell's ability to sense and indeed move up the chemical gradient. Thus we couple the cell's internal state to an external chemoattractant to investigate if the key processes of osmotic pressure extension and $\mathrm{Ca}^{2+}$ controlled contraction are sufficient to produce chemotaxis. We show, by choosing suitable functional forms for the spatial chemoattractant profile, that the model exhibits sustainable cell motion in a two dimensional scenario. Furthermore, we find that the model displays chemotactic sensitivity to the external chemical concentration and actively migrates toward the highest concentration. Also, the application of an asymmetric profile for the chemoat- tractant over the spatial domain of the cell produces a corresponding asymmetric local cellular deformation, which is so characteristic of cell behaviour. We present quantitative data for the mean velocity of the cell which is consistent with experiment. Obviously, due to the complexity of cellular processes involved, some simplifying assumptions and approximations must be made. However, the essential ingredients of the system are not sacrificed. This system allows one to see how the contemporary model of cellular motion is sufficient to produce motion in a two dimensional domain and how, with appropriate coupling to the membrane, the model can produce chemotactic sensitivity and lamellae extension.

\section{THE MODEL EQUATIONS}

We conceptualise the cell (see Figure 4) as a deformable material which is equipped with a polygonal boundary. That is the boundary is continuous and piecewise differentiable. The cell domain is parti- 
tioned, using triangular elements, so that the variables of the system may be specified at the nodal points. Evolution of the system will involve displacements of the cellular material so that we have a moving boundary problem. Additionally, the moving domain of the cell will be coupled to a fixed mesh underlying the cell where we specify external spatial quantities explicitly. Here we can prescribe the concentration of chemoattractant in the substratum.

Since we are modelling a thin slice through the lamellipod we adopt a plane stress assumption in the equations of visco-elasticity.

The vector $\mathrm{u}(\mathrm{x}, t)=(u(\mathrm{x}, t), v(\mathrm{x}, t))$ denotes the displacement of a point $x$ in the cell If $x_{0}$ is the original position of a point on the cell, $\mathrm{x}_{1}$ the position at time $t$, then the displacement $\mathrm{u}\left(\mathrm{x}_{0}, t\right)=\mathrm{x}_{1}-\mathrm{x}_{0}$.

Let $c(\mathrm{x}, t)$ denote the concentration of calcium ions at spatial position $\mathrm{x}$ at time $t$.

These variables allow us to describe the state of the cell in terms of displacements from original configurations and also by the concentration of intracellular calcium $\mathrm{Ca}^{2+}$.

\section{CALCIUM CONSERVATION EQUATION}

Employing the law of mass conservation [30], we can examine the corresponding evolution of the calcium concentration in a region as follows

$$
\frac{\partial c}{\partial t}=-\nabla \cdot \mathrm{J}+P(n, c, x, t),
$$

where J denotes the flux transport of the calcium and $P$ appropriate production and loss terms modelled as follows. We assume a classical diffusion process, so that we have

$$
\mathrm{J}=-D_{c} \nabla_{c},
$$

$P$ comprises of terms which model the calcium kinetics within the cell. As stated previously, an influx of calcium through the cell membrane initiates the release of internal stores of calcium stored within various organelles. This is an autocatalytic process and is known as calcium-stimulated calcium release [30] or calcium-induced-calcium-release [18]. When the calcium concentration in the cytosol is above a certain threshold $c^{*}$ it stimulates the release of the internal stores. This behaviour may be modelled by the function given by

$$
\frac{\alpha c^{2}}{1+\beta c^{2}},
$$

where $\alpha, \beta$ are positive constants. These parameters correspond to the magnitude of the release and account for some limiting process repectively. We assume a linear resequestration of the calcium with uptake parameter $\lambda[36],[30]$ to give the total calcium kinetics $R(c)$ as

$$
P(c)=\frac{\alpha c^{2}}{1+\beta c^{2}}-\lambda c
$$

Although the exact biochemical details of the process are not fully understood, the qualitative features of these kinetics are represented in Figure 5. It has been shown [30] that the kinetic term $P(c)$ has three steady states when $4 \beta \delta^{2}<\alpha^{2}$, that is, the zero state, an unstable state at $c^{*}$ and a stable state at $c^{* *}$. When $4 \beta \delta^{2}>\alpha^{2}$ only the stable zero state exists. Furthermore, subject to initial conditions, it can be shown travelling wave solutions exist. In this paper, although both parameter regimes were examined, we provide no analytical results. This is largely due to the nature of the boundary conditions which we shall shortly describe.

Thus equation (1) becomes

$$
\frac{\partial c}{\partial t}=D_{c} \nabla^{2} c+\frac{\alpha c^{2}}{1+\beta c^{2}}-\lambda c .
$$

The authors in [36] considered the system as dependent only upon the calcium concentration and visco-elastic parameters alone. We now develop a two dimensional force balance equation based on that proposed in [36].

\section{FORCE BALANCE EQUATION EQUATION}

To address the mechanical force interactions within the material of the cell, we utilise the equations of visco-elasticity [25]. Visco-elastic models are reasonable candidates to describe small deformations of cells which possess fluid and elastic properties [14]. 


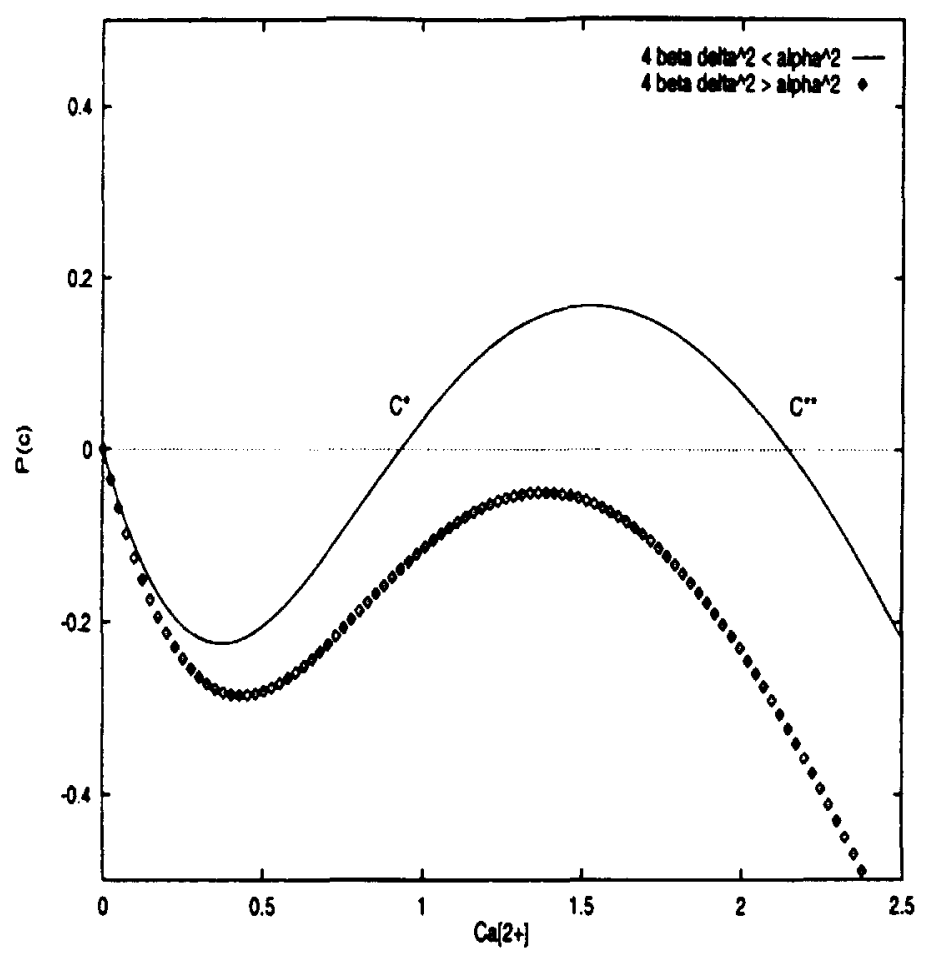

FIGURE 5 Qualitative features of the calcium release and uptake function $R(c)$. When the parameters $\alpha, \beta$ and $\delta$ satisfy the condition $4 \beta \delta^{2}<$ $\alpha^{2}$ the kinetics have 3 steady states. When $4 \beta \delta^{2}>\alpha^{2}$ only the zero steady state exists

This description leads us to consider an equation of the form

$$
\nabla \cdot \sigma+\mathrm{F}=0
$$

where $\sigma$ denotes the stress tensor, whose components are governed by the visco-elastic properties of the cell material and also allows inclusion of the intrinsic stresses developed in the cell. F denotes body forces or external forces which act on the cell.

Typical velocities of motile cells such as fibroblasts, epitheliocytes and leukocytes lie in the range $0.2 \mu \mathrm{m} / \mathrm{min} \longrightarrow 12 \mu \mathrm{m} / \mathrm{min}$ [6], [4], (refer Figure 6). The motion therefore is sufficiently slow so that inertial effects may be ignored [36], [30].

Contributions to the stress tensor $\sigma$ are from the visco-elastic aspects of the cell, and intrinsic terms from active traction and osmotic expansion. We may write the force balance equation as

$$
\begin{aligned}
& \nabla \cdot\left\{\left(\mu_{1}(c) \epsilon_{t}+\mu_{2}(c) \theta_{t} \mathrm{I}\right)\right. \\
& \left.\quad+\frac{E(c)}{1+v}\left(\epsilon+\frac{v}{1-2 v} \theta \mathrm{I}\right)+\sigma_{o}+\sigma_{a}\right\}=\sigma_{s},
\end{aligned}
$$

where the local deformation is given by the strain tensor [25] denoted by

$$
\epsilon=\frac{1}{2}\left(\nabla \mathrm{u}+\nabla \mathrm{u}^{T}\right) .
$$

Here, $E(c)$ denotes Young's modulus per unit volume, $v$ the Poisson ratio, $\mu_{1}(c), \mu_{2}(c)$ are shear and bulk viscosities respectively. $\theta=\nabla \cdot u$ denotes the dilation of the material. These values relate to the intrinsic properties of the cell. In reality, to define precisely these coefficients is itself a dynamical question in that they will continously vary with the cell's changing state. However, for simplicity we make the above parameters constants, thus the cell cytosol may 


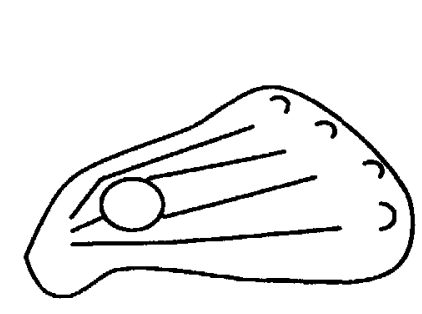

Speed

Adhesion

Fibroblast

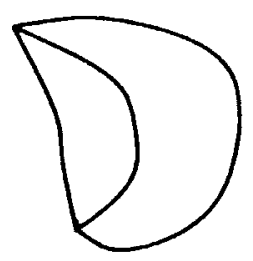

12

0.2

high

\section{Epitheliocytes}

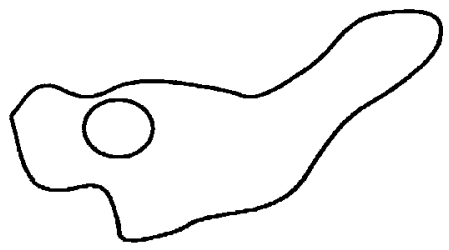

$>12$ fair

low

\section{Amoeba, Leukocytes}

FIGURE 6 Typical motile cells with their average speeds in $\mu \mathrm{m} / \mathrm{min}$. Redrawn from [6]

be approximated as a linear visco-elastic material, and we may describe small deformations in terms of the displacements.

The contribution from osmotic pressure is denoted by $\sigma_{o}$, and active traction by $\sigma_{a}$. The substrate forces to the cell are denoted by $\sigma_{s}$ which comprise of the external forces to the cell [6],[37]. In actual fact as we shall describe shortly this force will only occur as a boundary condition. Write the above as

$$
\begin{aligned}
\nabla \cdot\{ & \left(\mu_{1}(c) \epsilon_{t}+\mu_{2}(c) \theta_{t} \mathrm{I}\right) \\
& \left.+\frac{E(c)}{1+v}\left(\epsilon+\frac{v}{1-2 v} \theta \mathrm{I}\right)\right\}=\mathrm{F},
\end{aligned}
$$

where

$$
\mathrm{F}=-\nabla \cdot\left\{\sigma_{o}+\sigma_{a}\right\}+\sigma_{s}
$$

We shall now introduce forms for the components $\sigma_{o}, \sigma_{a}$ and $\sigma_{s}$. Using our simplifying assumption that the substrate provides no resistive forces, we have $\sigma_{s}=0$ at all points in the cell domain, except where the cell is attached to the substrate. These points will be manifest as boundary conditions imposed on the force balance equation. For example, during the extension phase the cell extends its lamellae, this indicates that substrate tethers have been broken. However, the rear portion of the cell remains fixed, thus implying substrate forces can be assumed greater than the extensile forces or that the tethers remain intact. We can therefore fix the cell at the basal region, evidently a zero Dirichlet condition on the displacements. We will specify the boundary conditions fully later. As mentioned earlier, haptotaxis may be incor- 


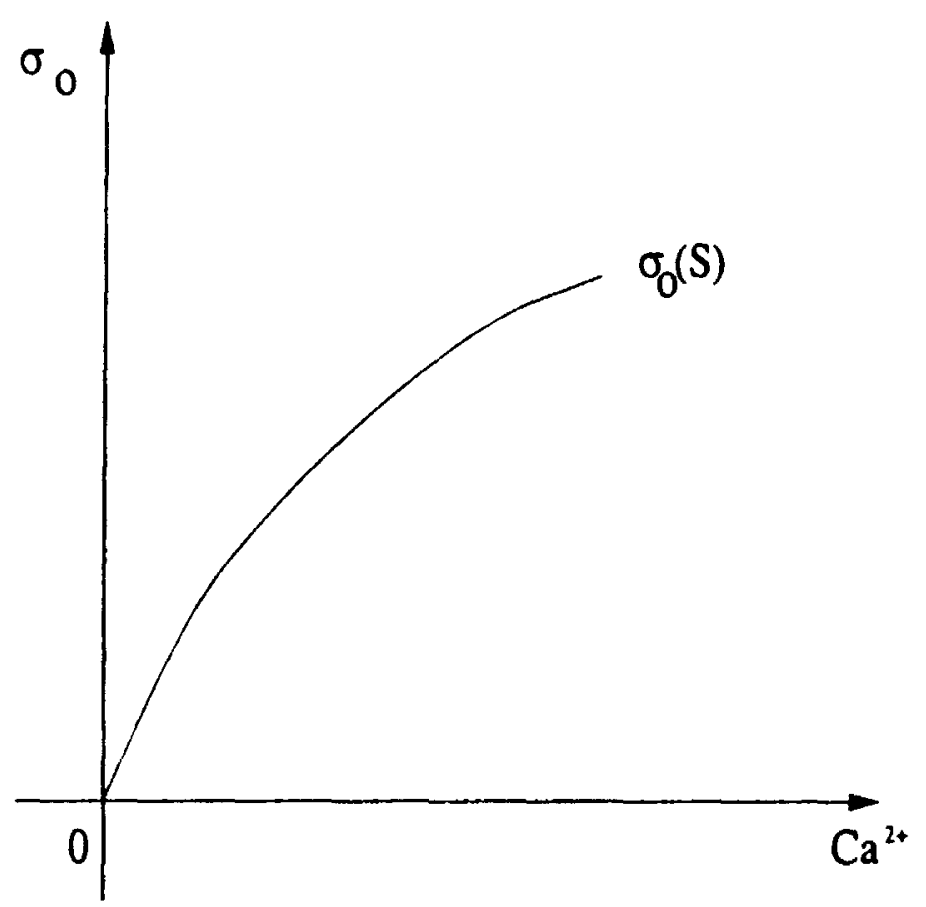

FIGURE 7 The influx and release of $\mathrm{Ca}^{2+}$ initiates solation of the cytogel. As the volume of disassembled cytogel $\mathrm{S}$ in the lamella increases, it reduces the elastic modulus of the cytogel. The lamella then extends due to the osmotic/hydrostatic swelling pressure [36]

porated into the system. The quantity $\sigma_{s}$ could be functionally related to some adhesion agent such as fibronectin which could be specified externally to the cellular domain. However, we shall not address haptotaxis in this model.

Figure 7 shows the qualitative features of osmostic pressure as presented in [36]. Osmotic pressure increases with increasing volume fraction of disassembled cytogel denoted by $S$. Since the state of the cytogel is dependent upon calcium concentration, we make the osmotic swelling pressure a function of calcium.

Based on Figure 7 we choose a simple linear form for $\sigma_{o}$ which designates that the osmotic pressure at a point within the cell is simply a linear function of calcium concentration. This relation indicates that an associated reduction in the elastic properties of the cell's apical region would accompany an influx of calcium $\mathrm{Ca}^{2+}$, which is a reasonable assumption. Thus we set

$$
-\nabla \cdot\left\{\sigma_{o}\right\}=\nabla \cdot\{\pi c \mathrm{I}\} .
$$

Since an outward force is generated, $\sigma_{o}$ has a postive coefficient. In vector form this becomes

$$
\nabla \cdot\{\pi c \mathrm{I}\}=\pi\left(\begin{array}{l}
\frac{\partial c}{\partial x} \\
\frac{\partial c}{\partial y}
\end{array}\right) .
$$

The active traction $\sigma_{a}$ generated by the cell must account for a directional bias. This bias must correspond in some way to the chemotactic agent in order for the cell to migrate. Many studies (see [3] for example) have used techniques such as immunofluorescence microscopy and have observed an alignment of actin stress fibres in the direction of the extending lamellae. We therefore assume that the level of polymerized actin is proportional to the $\mathrm{Ca}^{2+}$ concentration during influx. The maximum of orientated fibres would occur when the cell closes its membrane to further influx of $\mathrm{Ca}^{2+}$. Thus, we choose the gradient of the calcium profile at this time to specify in which direction the subsequent contraction force should operate. Thus, an influx and local release of 
calcium would initiate polymerization and alignment of the actin fibres in the profile of the calcium gradient. Once the cell closes the plasma membrane to further influx of $\mathrm{Ca}^{2+}$, then $\mathrm{Ca}^{2+}$ will be resequestered into internal organelles and depolymerization of the actin network will commence. This will generate contractile forces which will act throughout the cell due to the cell's cytoskeleton.

Thus at time $t=t_{e}$ we essentially capture the calcium gradient

$$
\nabla c\left(\mathrm{x}, t_{\mathrm{e}}\right)
$$

we set

$$
-\nabla \cdot\left\{\sigma_{a}\right\}=-\nabla \cdot\left\{\delta \nabla c\left(\mathrm{x}, t_{e}\right)\left(\int_{\Omega} \tau(c) d \mathrm{xI}\right\},\right.
$$

again in a two dimensional vector form this becomes

$$
-\nabla \cdot\left\{\sigma_{a}\right\}=-\delta\left(\begin{array}{l}
\frac{\partial c\left(\mathrm{X}, t_{e}\right)}{\partial x} \tau c \\
\frac{\partial c\left(\mathrm{X}, t_{c}\right)}{\partial y} \tau c
\end{array}\right),
$$

where

$$
\delta^{-1}=\sup \left|\nabla c\left(\mathrm{x}, t_{e}\right)\right| \quad \mathrm{x} \in \Omega,
$$

and $\tau$ a positive constant.

This provides the mechanism to specify the direction in which the active contraction operates. We assume a linear contractile force proportional to the calcium concentration is generated, given by $\tau c$ whose direction is specified by $\nabla c\left(\mathrm{x}, t_{e}\right)$. The value chosen for the parameter $\delta$ is such that the product $\delta \nabla c\left(\mathrm{x}, t_{e}\right)$ is normalized and has maximum value not exceeding unity. During the contractile phase we assume the cell has sufficient attachments at the apical region, this provides a boundary condition to fix the cell so that the contractions pull the cell body in the direction of the contraction. This is a necessary condition otherwise the cell would be unable to move. We shall discuss boundary conditions shortly.

The term F may be considered as a forcing component to the cell material provided by osmotic/hydrostatic pressure and cellular traction. We consider the cell to be on a substrate which has a distribution of some chemical attractant or adhesion modifying complex which will influence the cell's preferred orientation and direction of motion. [49], [48]. This could be realised in vivo by a cell sensing an activating agent, for example an endothelial cell sensing some tumour angiogenesis factor (TAF).

Introducing the following nondimensionalizations

$$
\begin{aligned}
& \mathrm{u}^{*}=\frac{\mathrm{u}}{L}, \quad x^{*}=\frac{x}{L}, \quad y^{*}=\frac{y}{L}, \\
& t^{*}=\frac{t}{T}, \quad c^{*}=\frac{c}{c_{0}}, \quad d_{c}=T D_{c},
\end{aligned}
$$

gives

$$
\begin{gathered}
\nabla^{*}=L \nabla, \quad \alpha^{*}=\operatorname{Tac}_{0}, \quad \beta^{*}=\beta c_{0}^{2}, \\
\mu_{1}^{*}=\frac{\mu_{1}}{E T}, \quad \mu_{2}^{*}=\frac{\mu_{2}}{E T}, \\
\tau^{*}=\frac{\tau c_{0}}{E}, \quad \pi^{*}=\frac{\pi c_{0}}{E}, \quad \lambda^{*}=\lambda T .
\end{gathered}
$$

Dropping the asterisks for notational simplicity, the above system becomes:

$$
\begin{gathered}
\frac{\partial c}{\partial t}=d_{c} \nabla^{2} c+\frac{\alpha c^{2}}{1+\beta c^{2}}=-\lambda c \\
\nabla \cdot\left\{\left(\mu_{1} \epsilon_{t}+\mu_{2} \theta_{t} \mathrm{I}\right)+\frac{1}{1+v}\left(\epsilon+\frac{v}{1-2 v} \theta \mathrm{I}\right)\right\}=\mathrm{F}, \\
\mathrm{F}=\nabla \cdot\{\pi c \mathrm{I}\}-\left\{\delta \nabla c\left(\mathrm{x}, t_{e}\right) \tau c \mathrm{I}\right\} .
\end{gathered}
$$

For equation (4) and equation (5) we prescribe the following initial conditions

$$
\mathrm{u}(\mathrm{x}, 0)=0, \quad c(\mathrm{x}, 0)=0, \quad \mathrm{x} \in \Omega .
$$

This merely states there is initially zero displacement of the cellular material and that the intracellular concentration is at its unstimulated level. Thus on completion of each extension-contraction phase the state variables $c(\mathrm{x}, t)$ and $\mathrm{u}(\mathrm{x}, t)$ have to these initial configurations.

To complete the specification of the model equations we must define the boundary conditions which play a principal role. Before we define the boundary conditions, recall the extension phase and contraction phase time periods are given in the following definitions. The extension phase, is defined as time period given by the set $T_{e}=\left(t \in \mathrm{R}: 0 \leq t \leq t_{e}\right)$ and the combined contraction/relaxation period is defined for the set $T=\left(t \in \mathrm{R}: t_{e}<t\right)$. Let $\partial \Omega_{l}$ be the portion of the cell boundary at the leading edge which is receptive to external compounds.

Similarly, let $\partial \Omega_{b}$ be the cell boundary at the basal region of the cell. The lateral edges of the cell are denoted by $\partial \Omega_{s}$. The total boundary of the cell being 


\section{x及 - boundary}

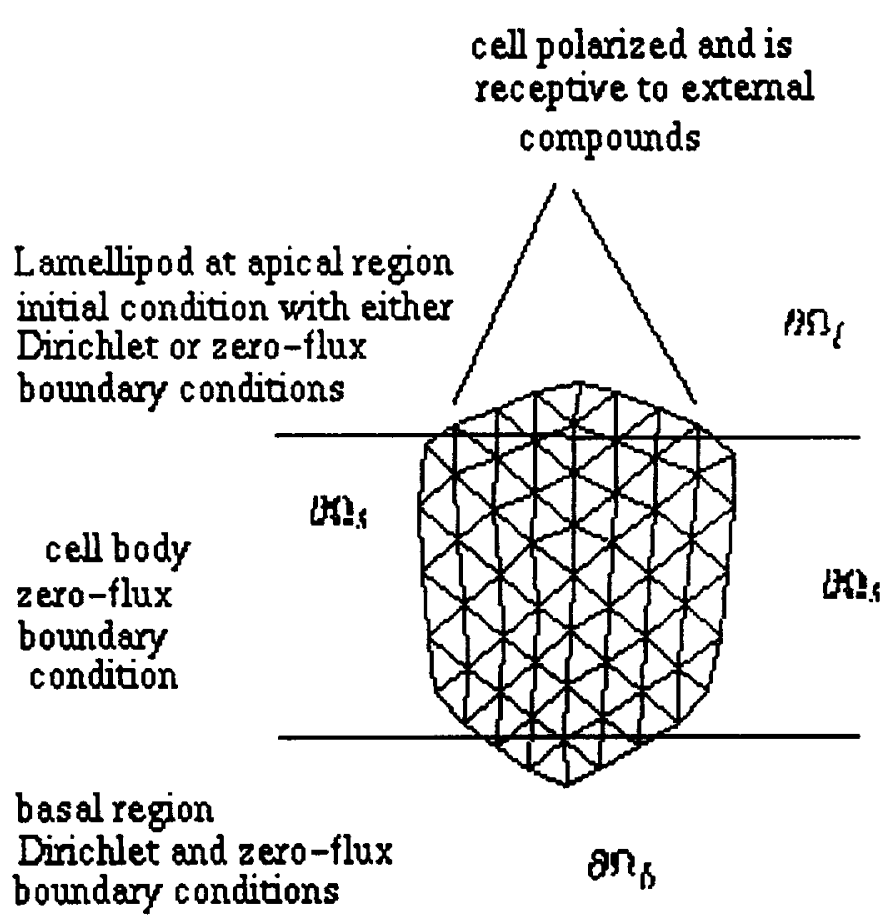

FIGURE 8 Finite element mesh of a cell with associated boundary conditions. The leading edge of the cell $\partial \Omega_{1}$ is permeable to the influx of $\mathrm{Ca}^{2+}$ during the extension phase. The basal $\partial \Omega_{\mathrm{b}}$ and lateral $\partial \Omega_{\mathrm{s}}$ regions during this phase are impermeable, hence a zero flux condition is imposed. Also, during the extension phase, the leading edge and cell body are free to deform whereas the basal region is assumed fixed, thus we have $u:=0$ on $\partial \Omega_{\mathrm{h}}$. Similar conditions apply during the contraction phase. See main text for full details

$\partial \Omega$ refer Figure 8. At the leading edge $\partial \Omega_{l}$ of the cell we will establish a relation between the permeability of the membrane and hence the transport of $\mathrm{Ca}^{2+}$ across the membrane during the extension phase. The remaining regions $\partial \Omega_{b}$ and $\partial \Omega_{s}$ of the cell we will assume are impermeable to influx. We now specify the boundary conditions for equation (4) at the leading edge of the cell for the time period $T_{e}$

In [36] the boundary condition is given by

$$
d_{c} \nabla c\left(\partial \Omega_{l}, t\right) \cdot \mathbf{n}=K\left(c_{e x}-c\left(\partial \Omega_{l}, t\right)\right) .
$$

Where $K$, a positive constant, relates to the membrane permeability, $c_{e x}$ the external calcium concentration and $d_{c}$ the calcium diffusion parameter described earlier.

$$
d_{c} \nabla c\left(\partial \Omega_{l}, t\right) \cdot \mathrm{n}+K c\left(\partial \Omega_{l}, t\right)=K c_{e x} .
$$

Where $\mathbf{n}$ is an outward unit normal to the boundary. Also, typically $d_{c} \ll<1$ and so the first term in this expresssion is negligible, we accordingly approximate this condition to

$$
c\left(\partial \Omega_{l}, t\right)=c_{e x} \quad t \in T_{e} .
$$

In order for the cell to display chemotactic behaviour, it must sense the chemotactic agent in the surrounding environment and utilise this information for directed motion toward the source. As discussed earlier, the mechanism for motion is governed by the influx and local release of calcium. Clearly then we require a method to relate the spatial distribution of some chemotactic agent to the level of calcium which may penetrate the cellular membrane.

This is achieved as follows: Firstly, we provide a spatial function $h(\mathrm{x})$. This gives us the facility of 


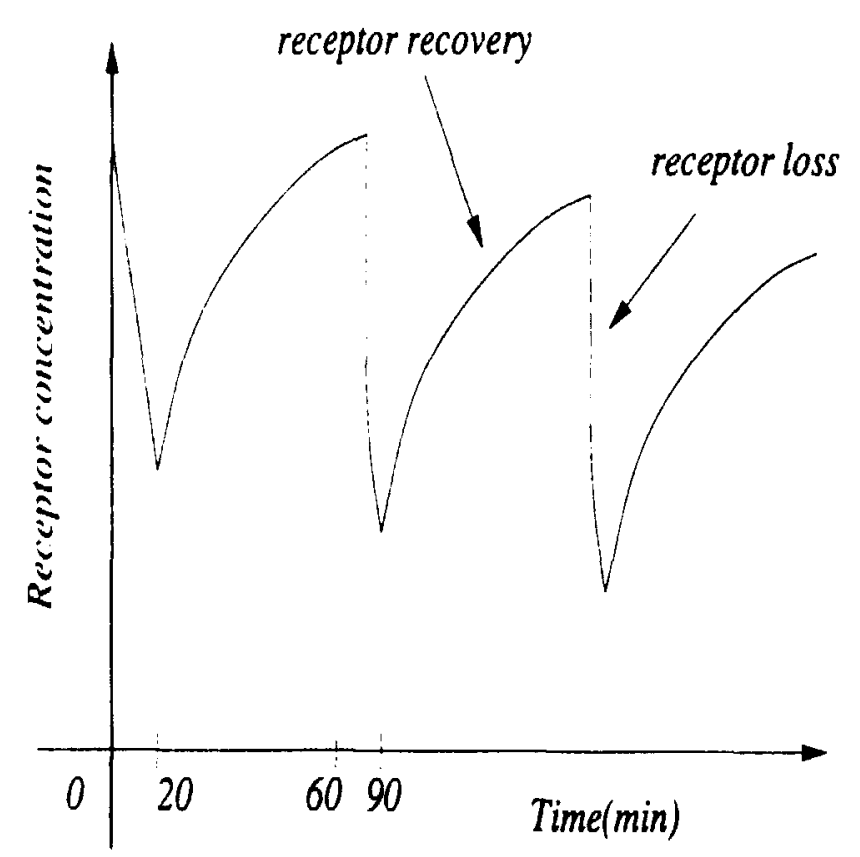

FIGURE 9 Chemotactic peptide receptor recovery-loss cycle. The concentration of chemoattractant affects the permeability of the cell membrane. The receptors are internalised when the chemoattractant saturates and expressed after a relaxation period. Redrawn from [49]

ascribing an external chemotactic profile. Also, in [49] chemotactic peptide receptors on the cell membrane were found to have a recovery-loss cycle, refer Figure 9. Since these receptors govern the permeability of the cell membrane, it seems reasonable include a qualititative feature of this effect at the leading edge boundary. Thus, an influx of calcium through the membrane will be modulated by the external chemotactic profile and by the receptor recovery state. Hence, we set the calcium concentration on the boundary $c\left(\partial \Omega_{l}, t\right)$ to be a product of the external chemotactic concentration $h(\mathrm{x})$ and $\sin (\pi t)$ for $t \in T_{e}$ to express the stimulatory profile.

$$
c\left(\partial \Omega_{l}, t\right)=\sin (\pi t) h(\mathrm{x}), \quad t \in T_{e} .
$$

On the remaining boundary of the cell, namely $\partial \Omega_{b}$ the basal region and lateral $\partial \Omega_{s}$ edges of the cell, we specify zero flux or Neuman conditions during the the time interval $T_{e}$. This corresponds to the cell membrane being impermeable to calcium on these portions of the cell membrane. Thus we have

$$
\begin{aligned}
& \nabla c\left(\partial \Omega_{l}, t\right) \cdot \mathrm{n}=0, \quad t \in T_{e} . \\
& \nabla c\left(\partial \Omega_{b}, t\right) \cdot \mathrm{n}=0, \quad t \in T_{e} .
\end{aligned}
$$

For $t>t_{e}$ i.e. the contraction/relaxation time period we assume the lamellipod membrane has closed its channel pathways and so further influx of calcium ceases. Again as the membrane becomes non-porous this translates to a zero-flux condition in mathematical terms. Thus, the entire boundary $\Omega$ has a zero flux condition and the cell is closed to its environment, and we have

$$
\nabla c(\partial \Omega, t) \cdot \mathrm{n}=0, \quad t>t_{e} .
$$

The boundary conditions for the displacements are described as follows Firstly for the extension period $t$ $\in T_{e}$

$$
\mathrm{u}\left(\partial \Omega_{b}, t\right)=0, \quad t \in T_{e} .
$$

This states the cell is fixed at the basal region. Here we assume that the cell maintains adherence in this region, whereas in the lamellipod, the cell is free to deform, thus having a free boundary. 
TABLE II

\begin{tabular}{cccc}
\hline Parameter & Numeric range & Units & Reference \\
\hline Traction per cell $\tau$ & $0.03-0.27$ & dynes $/$ cell $^{2}$ & {$[21]$} \\
Young's modulus $E$ & $1-10^{3}$ & dynes $/ \mathrm{cm}^{2}$ & {$[1],[45]$} \\
Poisson ratio $v$ & $0-0.5$ & $\mathrm{~cm} / \mathrm{cm}$ & {$[25]$} \\
Viscosity $\mu$ & $10^{2}-10^{5}$ & poise & {$[45],[13]$} \\
Average cell length $L$ & $20-100$ & $\mu \mathrm{m}$ & {$[13],[43]$} \\
Extension time $T_{e}$ & $6 \times 10-4 \times 10^{3}$ & secs & {$[13],[43]$} \\
Contraction time $T$ & $6 \times 10-6 \times 10^{3}$ & secs & {$[13],[43]$} \\
Osmotic pressure $\pi$ & $4 \times 10^{2}$ & Torrs & {$[37]$} \\
Calcium $C a^{2+}$ uptake $\lambda$ & 10 & sec $^{-1}$ & {$[10][18]$} \\
Calcium $\mathrm{Ca}^{2+}$ diffusion $D_{c}$ & $2.25 \times 10^{2}$ & $\mu \mathrm{m}^{2} / \mathrm{sec}^{2}$ & {$[101[18]$} \\
\hline
\end{tabular}

During the contraction/relaxation phase $t \in T$ at the leading edge $\partial \Omega_{l}$ we have a reversal of this situation to get

$$
\mathrm{u}\left(\partial \Omega_{l}, t\right)=0, \quad t>T_{e} .
$$

Physically, this corresponds to the lamellipod achieving full adhesion to the substrate and becoming fully stabilised due to the actin network. The subsequent cellular contraction overcomes substrate tethers and so the basal region has a free boundary.

It remains for us to establish what is the duration of the time periods $T_{e}$ and $T$. We propose to use experimentally observed extension periods $T_{e}$ gained from papers such as [43], and to allow the calcium kinetics in the interior of the cell to determine the time period $T$. That is to allow contraction to continue until the calcium has returned to a zero state.

Equation (4) and equation (5), together with the boundary conditions equation (7) - equation (12) provide an idealised model of the cell's varying intracellular calcium concentration, and the associated viscoelastic deformations.

\section{ESTIMATION OF PARAMETERS}

A requirement for the model to be informative is that it is capable of reproducing real cellular behaviour within the correct parameter ranges. As such, where possible we use parameter values which have been experimentally verified. Solving the system (4) and (5), we seek quantitative and qualitative behaviour which corresponds to both experimentation and observation. Typical quantitative information which is known about cellular motion is cell speed thus we seek agreement in this fact. Similarly we would expect the total chemotactic response of the cell to culminate in the persistent motion toward the chemoattractant. Estimation of parameters is very difficult in the biological domain since empirical values can vary widely if they are available at all. Not only are

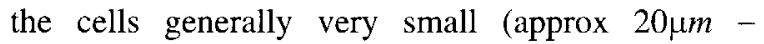
$120 \mu \mathrm{m}$ ), but slight variations in control experiments may cause significant changes in observable data.

We choose a characteristic length scale of $L=100 \mu \mathrm{m}$. This is the mean length of a non-muscle cell. We can then investigate cellular motion with respect to its length, since it is known cells can migrate up to their own length in approximately $60-80 \mathrm{mins}$ [3], [6] under optimum conditions. The role of actin plays an important role in cellular motion. Actin has a characteristic polymerization time of around $1 \mathrm{~min}$ (Zigmond 1993), and so the minimum time in which to detect an expansion or contraction of a cell is approximately $3 \mathrm{~min}$. We therefore choose our reference timescale to be $T=1 \mathrm{~min}$. Furthermore, we impose this fixed timescale to be the duration of the extension phase $T_{e}$, whereas the relaxation/contraction phase will be dependent upon the calcium kinetics within the cell. Thus, $T$ is the time taken for the cell to restore the calcium level to the initial state by resequestration or removal. Table II shows experimental or observed ranges for the parameters of the system. Using these values in the non-dimensional relations provides the appropriate noudimensional ranges. 
The calcium $\mathrm{Ca}^{2+}$ diffusion parameter $D_{c}$ and uptake $\lambda$ were drawn from [10] where a full parameter list and source can be found relating to a more extensive model of the calcium kinetics. We will discuss this point later in the text. The actual value of the diffusion is thought to vary for different cell types, the above values relate to muscle cell types. Since the primary function of the muscle cell is to respond directly to calcium concentration it is reasonable to expect that the same parameter values in non-muscle cells would be reduced.

\section{NUMERICAL SIMULATIONS AND RESULTS}

To solve the system of equations (4) and (5) we must correlate the intracellular calcium $\mathrm{Ca}^{2+}$ concentration at each time step with an associated forcing component. The generated force is calcium dependent and will be either an osmotic/hydrostatic extension force, or an actomysosin contractile force. The solution at each time step for the calcium concentration is dependent upon the boundary conditions (equations (7) - (12)). These boundary conditions enable calculation of the calcium influx and interior concentrations. Additionally, they prescribe which portions of the cell are fixed and which are free to deform at any given time in the cellular cycle. Once the intracellular calcium concentration has been established, we can calculate the corresponding effect upon the cellular material by solving equation (5). Thus we use the following algorithin

1. For time step $t+\delta t$ calculate values of calcium $c$ of intracellular calcium using equation (4) subject to boundary conditions. This will require the iterative solution of a non-linear system by Newton's method.

2. Use calculated values of calcium $c$ in the forcing components of force balance equation (5) until solution converges. The forcing component depends on the phase of the motion with either an extension or contraction phase to establish the appropriate boundary condition.
3. update nodal positions based on calculated displacements.

4. repeat from (1)

Thus the evolution of the intracellular calcium concentration drives the deformation of the cell material. The boundary conditions and particular movement phase imposed on the nodal positions of the cell will determine which nodes may be subject to displacement.

To solve such a moving boundary problem, it is convenient to adopt the Finite Element method which can accommodate irregular domains quite easily. Also this method was formulated with the solution of the equations of viscoelasticity in mind, thus our system is naturally suited to this method.

We solved the system of equations (5) and (4) and associated boundary conditions numerically using the Galerkin finite element method [12], [47]. We adopted linear triangular elemental forms for ease of implementation. The system was solved using Euler's method fully implicitly in time where convergence was achieved when a suitable criterion was satisfied. All matrix computations were solved using a pre-conditioned biconjugate gradient method since symmetric matrices were not guaranteed. Additionally, equation (4) is non-linear due to the release term, this demands an iterative solution at each time step. This was performed using Newton's method, where again a convergence criterion was imposed.

We now present the results of the model. Our demands of the model are to achieve agreement with qualitative and quantitative data. This would relate to cell speeds and to produce protrusions geometrically which will correspond to the existence of lamellipodia. We consider various hypothetical situtations where we are tracking, say an endothelial cell's motion which is sensing a chemotactic chemical. We impose several profiles for the chemoattractant which correspond to the function $h(\mathrm{x})$ in the model. In the following plots the kinetic term $P(c)$ has only the zero stable state, that is the parameters satisfy $4 \beta \delta^{2}>\alpha^{2}$.

Also we consider various initial geometric configurations for the cell. Firstly, consider Figure 10. This shows the simplest scenario for the chemoattractant profile, namely a constant value over the migratory 


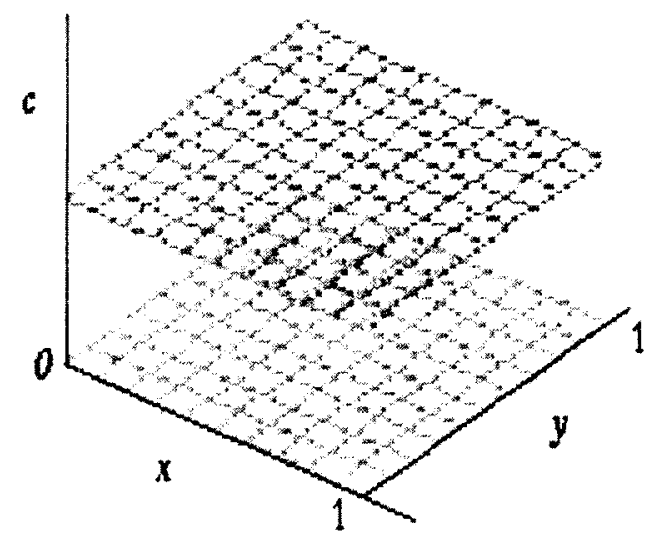

FIGURE 10 Constant chemoattractant profile. The cell will experience no spatial variation in chemoattractant concentration. As such, a symmetric cell will migrate straight ahead

domain. As such we would expect the cell to move in the direction of its initial orientation since it would sense the chemical but detect no spatial variation across its body. This is verified in Figure $11-14$, which clearly shows the cell migrating. Also note the basal region of the cell becomes more elongated with time since the contractile force experienced in this region is less than in the lamellipod, physically one could identify this as a retraction fibre.

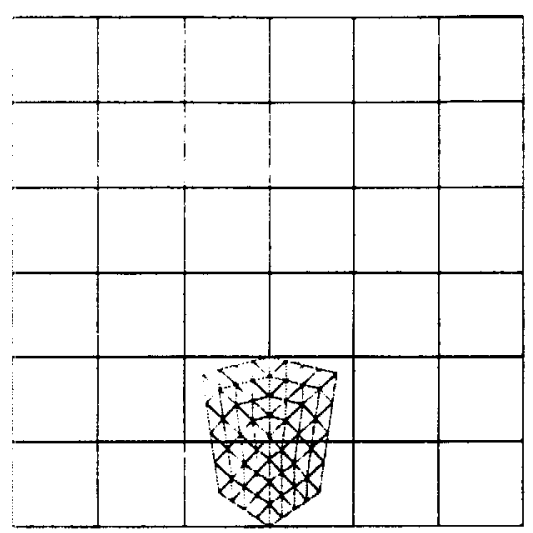

FIGURE $11 \mathrm{t}=0$

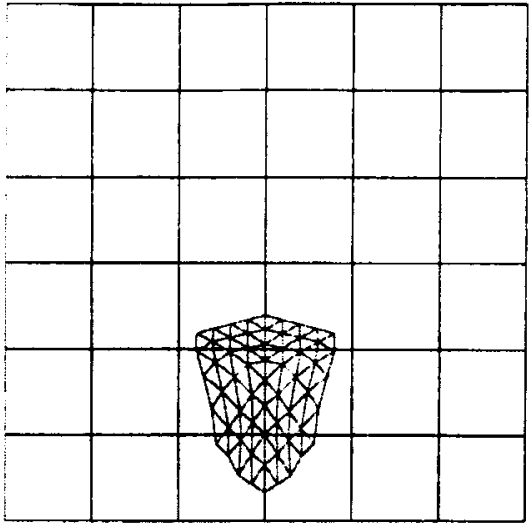

FIGURE $12 \mathrm{t}=15$

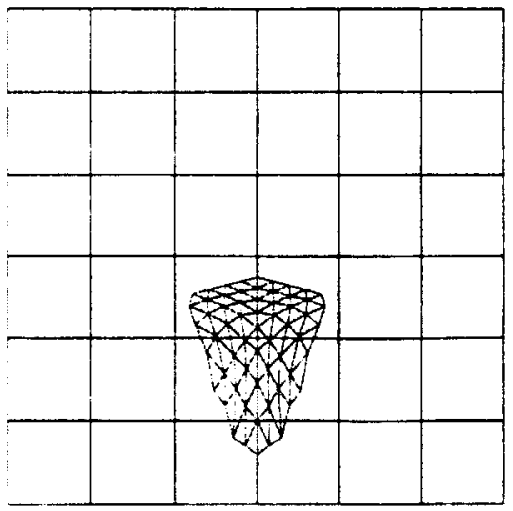

FIGURE $13 \mathrm{t}=30$

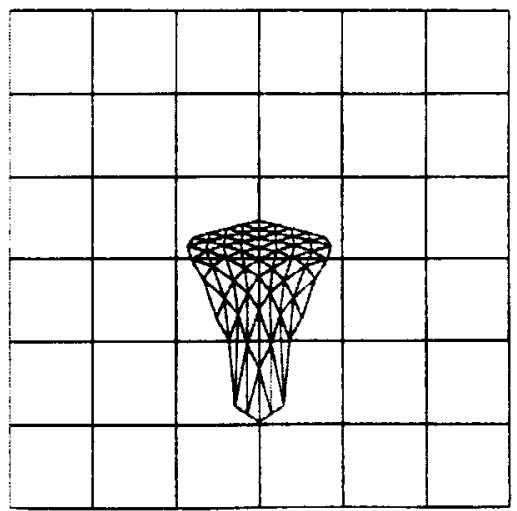

FIGURE $14 \mathrm{t}=45$ 


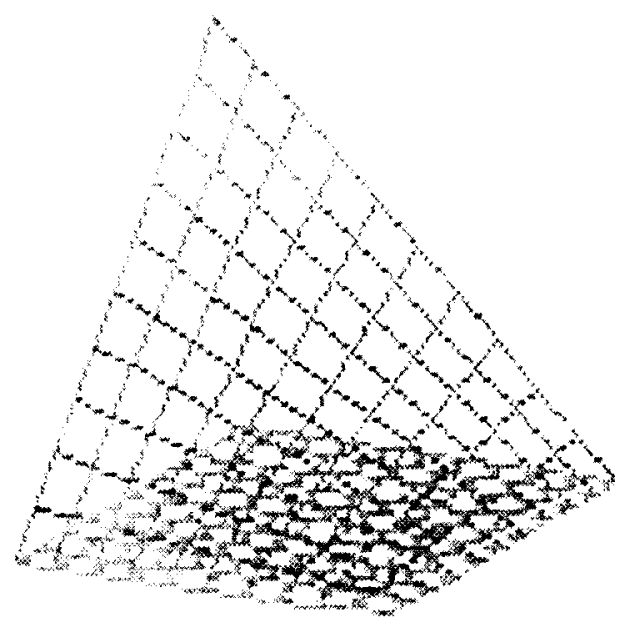

FIGURE 15 A linear chemoattractant profile provides a spatial incentive to the cell. The cell migrates up the chemical gradient

Choosing a constant chemoattractant profile may also correspond to the motile behaviour of fibroblasts in vitro. It is known that fibroblasts, when cultured [3] on a petri dish, will migrate in any direction and proliferate until they have colonised the whole dish and formed a complete monolayer culture. Thus, provided the environment has a sufficient calcium resource the above model would demonstrate this behaviour. Migration would only cease when external forces operate on the cell, this would be in the form of neighbouring cells and the petri dish walls.

Figure 15 shows the chemoattractant profile which we specified in order to stimulate the cell to changes in its direction of motion. The cell, sensing a gradient would actively migrate toward the highest concentration. Figure 16 - Figure 19 show the corresponding response.

On the cellular membrane many protrusions are frequently observed, this indicates the cell may be undergoing the cellular processes mentioned in different localities across its dimensions. To try to simulate this behaviour we imposed a chemoattractant profile as shown in Figure 20, and chose as our initial geometric configuration for the cell that shown in

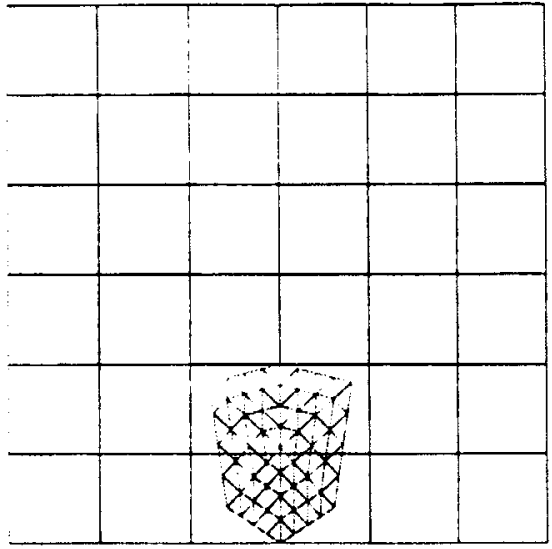

FIGURE $16 \mathrm{t}=0$

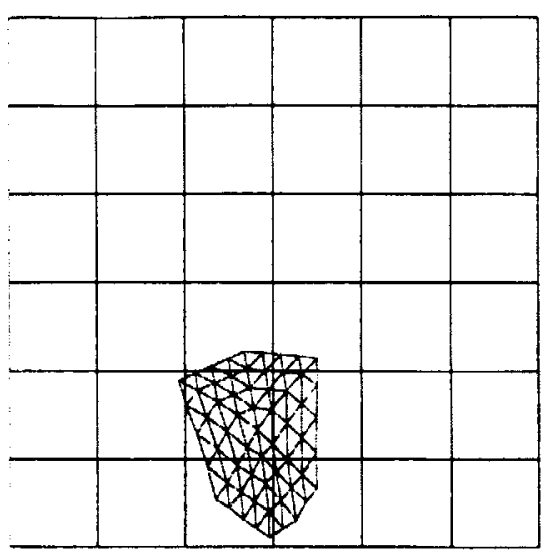

FIGURE $17 \mathrm{t}=15$

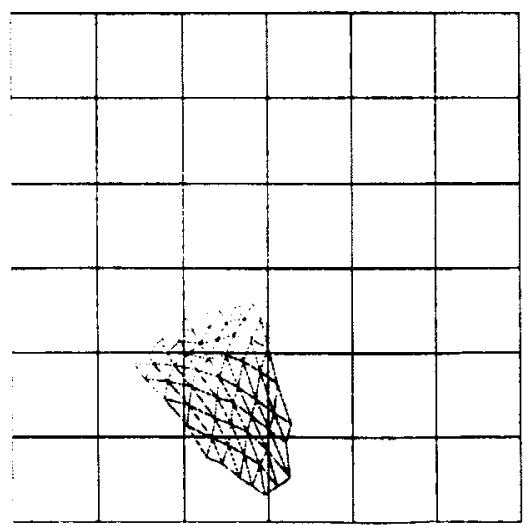

FIGURE $18 \mathrm{t}=30$ 


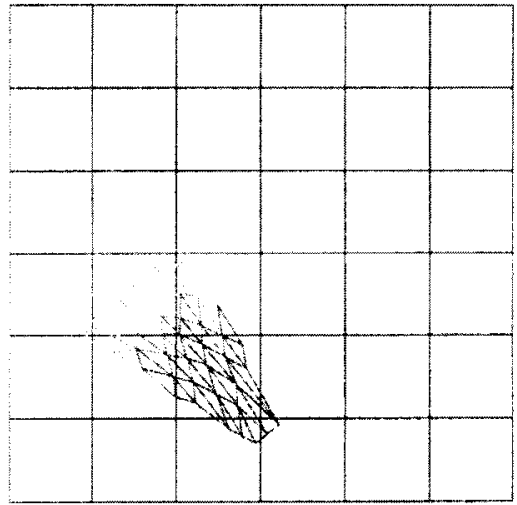

FIGURE $19 \mathrm{t}=45$

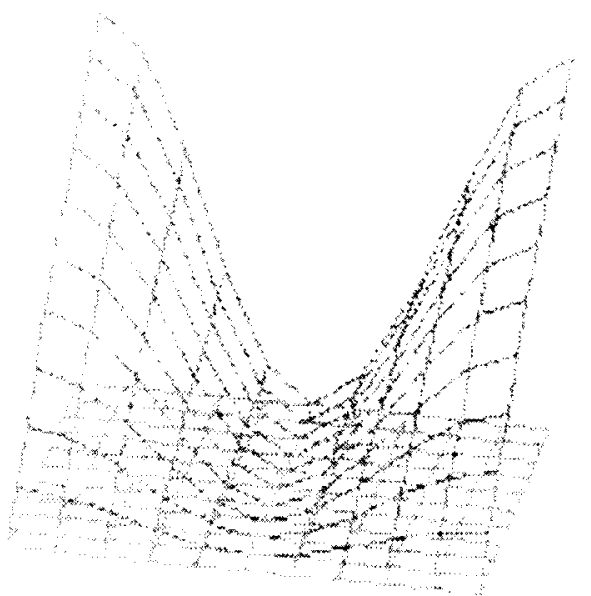

FIGURE 20 A cell sensing a cosine chemoattractant profile will tend to extend two lamellae

Figure 21. This configuration is one which may correspond to a typical epitheliocyte (see Figure 6).

Figures $21-24$ indicate the model is able to detect the spatial variation of the chemoattractant across the cellular dimension and shows the response to be of a chemotactic nature

Similarly Figures $26-27$ show the cell exhibiting rotation in response to the chemotactic profile in Figure 25. The cell senses two sources of attractant but responds more to the higher concentration. By vector summation of the forces generated by the sources the cell still makes forward motion.

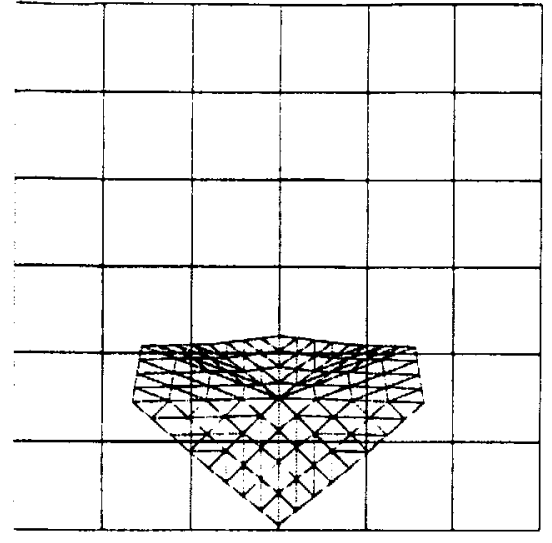

FIGURE $21 \mathrm{t}=0$

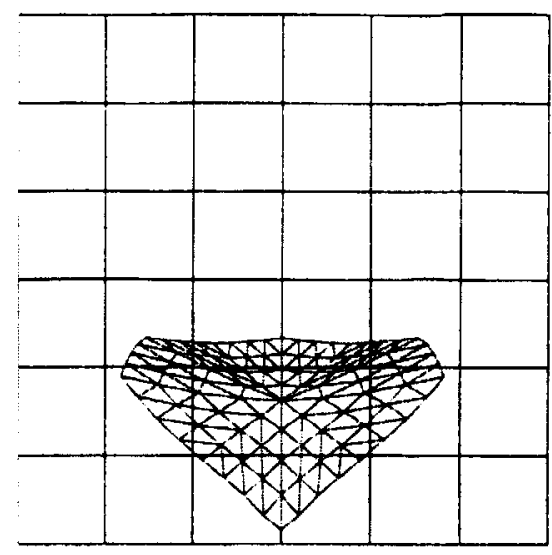

FIGURE $22 \mathrm{t}=2.5$

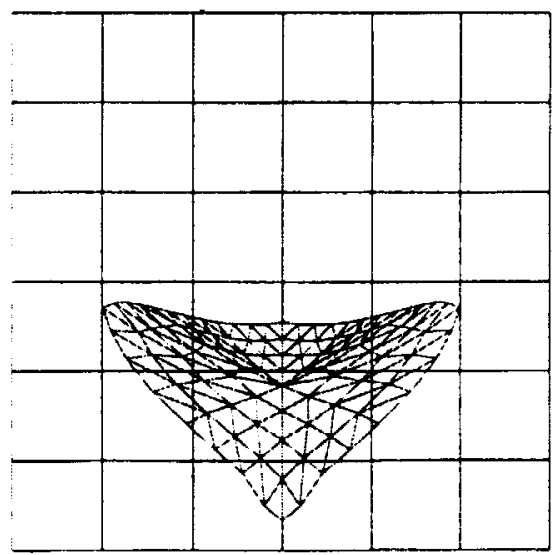

FIGURE $23 \mathrm{t}=7.5$ 


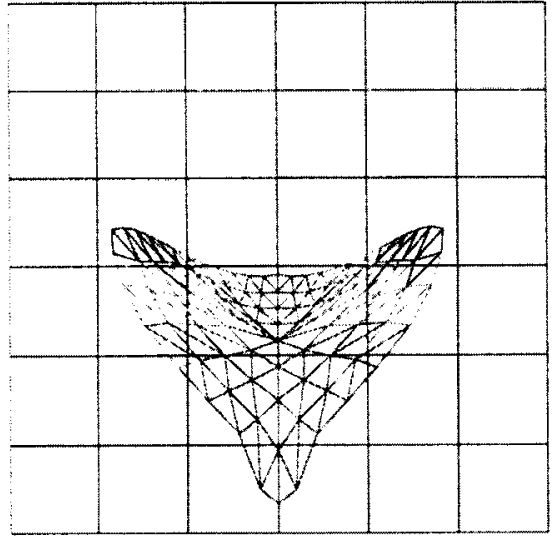

FIGURE $24 \mathrm{t}=10$

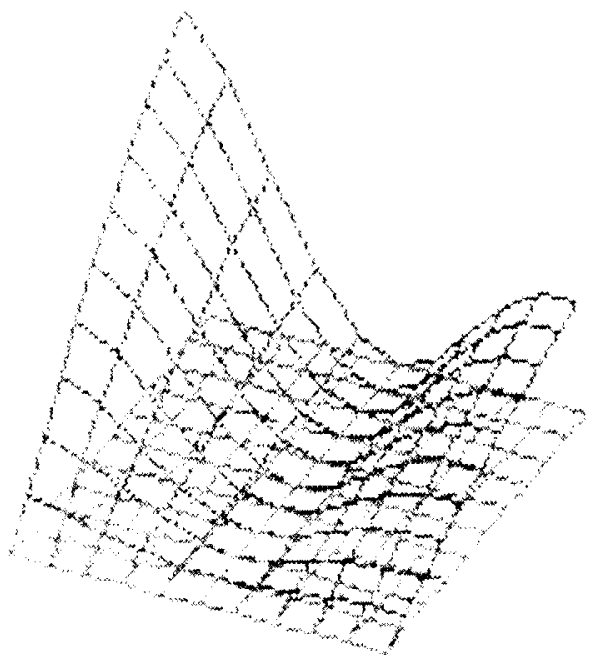

FIGURE 25 An asymmetric chemoattractant profile simulates behaviour shown in Figures 26 and 27

Next, to verify that the model produces cellular speeds of the correct order, we monitored the speed of the node which was initially located at the centroid of the cell, this provides us with the mean speed of the cell. Figure 28 shows the computed values. Upon rescaling to dimensional values we have speeds in the range $0-5 \mu \mathrm{m} / \mathrm{min}$ which is of the correct order.

As a further insight into the cellular kinetics, we monitored the calcium concentration at the leading

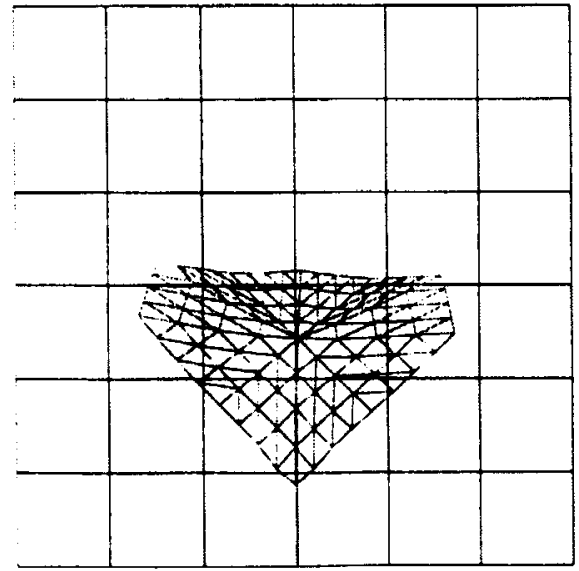

FIGURE $26 t=5$

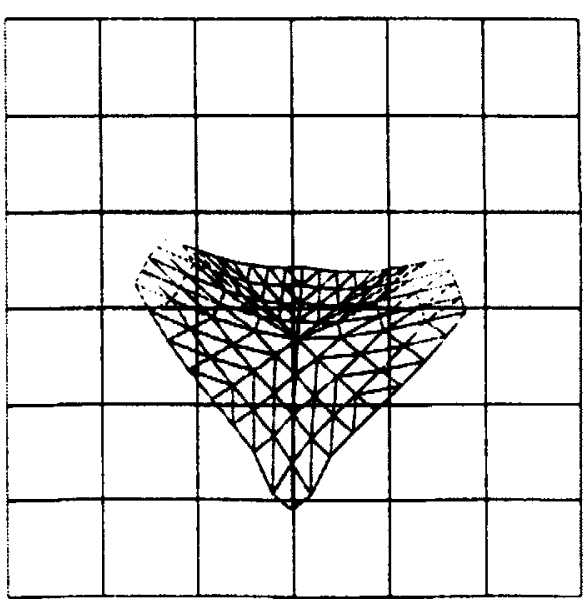

FIGURE $27 \mathrm{t}=10$

cellular membrane. Figure 29 shows the influx and uptake kinetics which the model produces. By varying the parameters $\alpha$ and $\lambda$ which relate to release and uptake of intracellular calcium, we can see the calcium kinetics directly influence the duration of the contraction/relaxation time period $T$. Figure 30 shows three differing contraction/relaxation time periods $T 1, T 2$ and $T 3$ as a result of varying release and uptake parameters. Here, we chose a parameter regime such that $4 \beta \delta^{2}<\alpha^{2}$. However, the system did not shift to the higher steady state $C^{* *}$ as similar models have dis- 


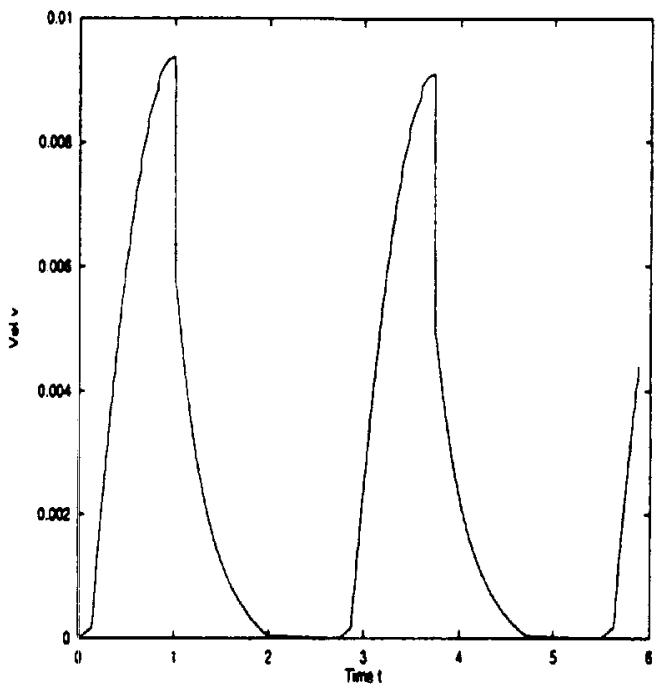

FIGURE 28 Associated mean velocity profile of the cell. The initial centre of mass of the cell is monitored to determine the mean velocity

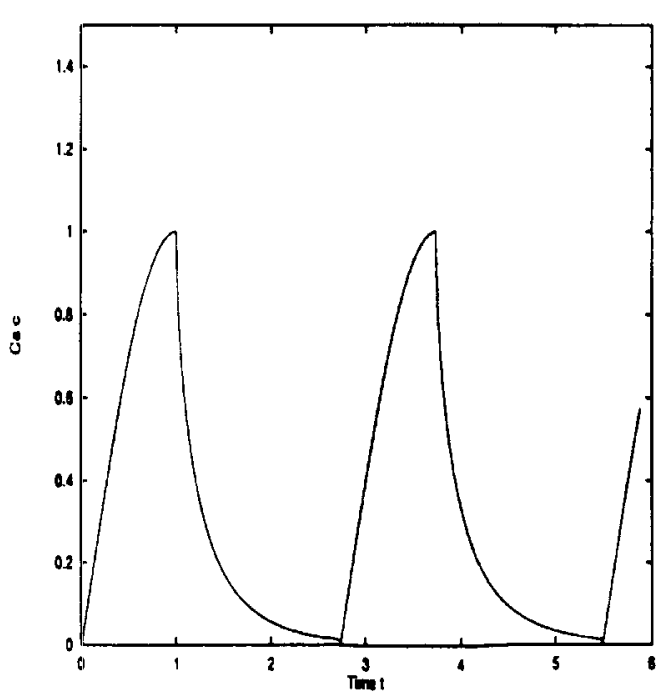

FIGURE $29 \mathrm{Ca}^{2+}$ at leading edge of cell. Chemoattractant molecules bind to the cell membrane receptors influencing the permeability of the cell wall

played [30]. This is probably due to the boundary condition and the initial condition providing insufficient perturbation to the system.

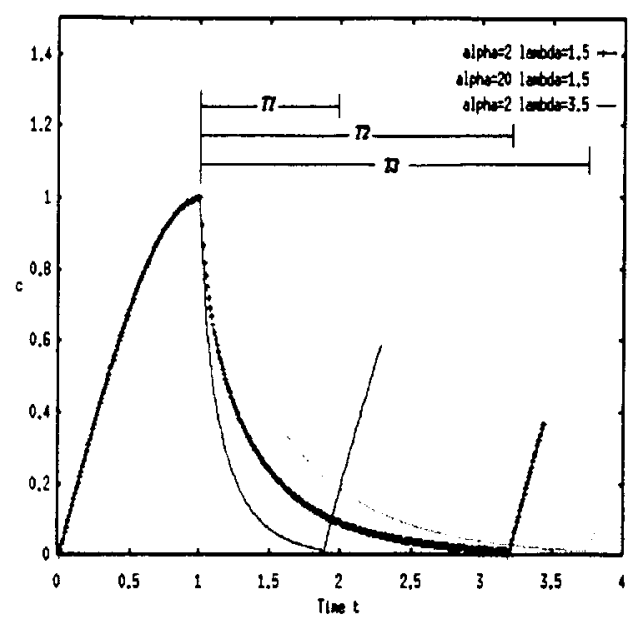

FIGURE $30 \mathrm{Ca}^{2+}$ at leading edge of cell. Effect of varying release $\alpha$ and uptake $\lambda$ parameters. T1,T2 and T3 denote different contraction/relaxation time periods

\section{DISCUSSION AND FUTURE WORK}

We have presented a simple two-dimensional mathematical model of a motile eucaryotic cell. The model correlates the force interactions between the viscoelastic properties of the cell against osmotic expansion or actomyosin mediated contraction. The forces generated from osmotic expansion and actomyosin contraction were given functional dependence on the intracellular $\mathrm{Ca}^{2+}$ calcium concentration. By utilising the calcium gradients established in the cell during calcium influx and intracellular release, we can specify the direction in which the expansion and subsequent contraction forces will operate. The primary orientation of these forces is in alignment with the maximum calcium influx. Since the cell membrane permeability may be governed by cell surface chemotactic receptors, the influx of calcium experienced by the cell, is associated with the external chemotactic profile. Translating the external chemotactic profile into an intracellular calcium gradient offers the potential of directional motion to the cell. Suitable application of boundary conditions which relate to cell-substrate adhesion provides the final ingredient for persistent cellular motion, furthermore, this 
motion exhibits chemotactic sensitivity. Variation in the chemotactic chemical over the cell body allows a corresponding variation in intracellular calcium which leads to the subsequent chemotactic response. Quantitatively, the model produces typical cellular velocities in the correct regime when using experimentally derived parameter values. On a qualitative level, the model can produce cellular protrusions which are commonly observed. Finally, by varying calcium uptake and release parameters, we can show how this may effect cell contraction times.

Clearly, given the complexity of the processes involved in cellular motion it is unreasonable to try and address all the aspects. However, by making the simplifying assumptions relating to the cell-substrate adhesion and membrane interactions, we have produced a model which captures the essential features. The model effectively is a minimal representation of a motile cell, and as such provides many avenues for enhancement. In principle, the treatment used in this model may be used to address the behaviour of small numbers of cells in response to some chemoattractant. This would not only be informative as to the spatial distribution of the cells due to local variation of chemoattractant, but also how, perhaps, intracellular events may influence that behaviour. This would be a novel approach in that it is neither a macroscpic model addressing large distributions of cells, nor focussing solely upon the intracellular variations. Future work could include;

- Investigate the behaviour of two or more cells.

- Couple the cell to the chemoattractant to simulate chemical uptake.

- Determine analytically if travelling wave solutions may exist with the above initial and boundary conditions.

- Incorporation of receptor-adhesion kinetics such as in [13], and link to the boundary conditions of the cell to provide a dynamic correspondence.

- Include strain activated calcium release [30].

- Non-constant visco-elastic parameters with functional relation to the calcium concentration.

- Membrane interaction with the cytoskeleton.
One immediate change which may be made to the model is the calcium kinetics. A more accurate description of the intracellular calcium concentration may be produced by considering the influx calcium and internally released calcium as two separate species [10], [18]. The model considered in [18] comprises of two coupled partial diffrential equations (one for influx and one for internally released calcium) to describe the total intracellular calcium concentration. The system is excitable in which travelling wave solutions arise. These solutions may be the source of the contraction waves which propagate across the cell, and may present interesting features in terms of the motion of the cell in a two dimensionl setting. Also, more sophisticated models may produce the spatial distributions of calcium which are observed in experiments [39].

\section{Acknowledgements}

The work of $\mathrm{M} \mathrm{J}$ Holmes was supported by an EPSRC Ph. D studentship.

\section{References}

[1] Adams D S. Mechanisms of Cell Shape Change: The Cytodynamics of Cellular Response to Chemical and Mechanical Loading. J Cell Biology. Vol. 117 (1), 83-93, 1992.

[2] N Akkas N (Ed). NATO ASI Series. Biomechanics of Active Movement and Division of Cells. Vol. 84, 285-328, 1994.

[3] Alberts B, Bray D, Lewis J, Raff M, Roberts K, Watson J. Molecular Biology of the Cell. 3rd Ed, 1994.

[4] Alt W, Dembo M. Cytoplasm Dynamics and Cell Motion: Two-Phase Flow Models. Sonderforschungsbereich 256 no. 541. Rhienische Friedrich-Wilhelms-Universitat Bonn, 1998.

[5] Alt W, Deutsch A (Eds). Dynamics of Cell and Tissue Motion. Birkhäuser Verlag, Berlin, 1997.

[6] Bereiter-Hahn J, Luiters H. The Role of Elasticity in the Motile Behaviour of Cells.

[7] Bromner-Frazer M. Alterations in the Neural Crest. $J$ Cell Biology. Vol. 101, 610-617, 1985.

[8] Carter S B. Principles of cell motility: The Direction of Cell Movement and Cancer Invasion. Nature. Vol. 208, $1183-$ $1187,1965$.

[9] Chaplain M A J, Giles S M, Sleeman B D, Jarvis R J. A Mathematical Analysis of a Model for Tumour Angiogenesis. J. Math. Biol. Vol. 33, 744-770, 1995.

[10] Chopra G C, Sleeman B D, Brindley J, Knapp D G. Velocity and Stability of Solitary Planar Travelling Wave Solutions of Intracellular $[\mathrm{Ca}]^{2+}$. Bulletin of Mathematical Biology. Vol. 61, 273-301, 1999.

[11] Dembo M, Harlow F H, Alt W. The Biophysics of Cell Surface Motility.

[12] Dhatt G, Touzot G. The Finite Element Method Displayed. John Wiley and Sons, 1984. 
[13] Dimilla P A, Barbee K, Lauffenberger D A. Mathematical Model for the Effects of Adhesion and Mechanics on Cell Migration Speed. Biophysical Journal. Vol. 60, 15-37, 1991.

[14] Dong C, Skalak R, Sung K L P, Schmid-Schonbein G W, Chien S. Passive Deformation Analysis of Human Leukocytes. J. Biomech. Eng. Vol. 110, 27-36, 1988.

[15] Dowben R M, Shay J W. Cell and Muscle Motility. Vol. 1, Plenum Press New York and London, 1981.

[16] Dunn G A, Brown A F. Alignment of Fibroblasts on Grooved Surfaces. Journal of Cell Science. Vol. 83, 313-340, 1986.

[17] Dunn G A, Zicha D. Dynamics of Fibroblasts Spreading. $J$ Cell Science. Vol. 108, 1239-1249, 1995.

[18] Dupont G, Goldbeter A. Properties of Intracellular Calcium Waves Generated by a Model Based on Calcium-Induced-Calcium-Release. J Biophysics. Vol. 67, 2191-2204, 1994.

[19] Harris A K. Cell Surface Movements Related to Cell Locomotion. Locomotion of Tissue Cells. Ciba Fdn Synp. 14, 3 26, 1973. Amsterdam, Holland.

[20] Harris A K, Dembo M. Motion of Particles Adhering to the Leading Lamella of Crawling Cells. Journal of Cell Biology. Vol. 91, 528-536, 1981

[21] Harris A K, Stopak D, Wild P. Silicone Rubber Substrata: A New Wrinkle in the Study of Cell Locomotion. Science. (Wash D. C) Vol. 208, 177-179, 1980.

[22] Harris A K, Stopak D, Wild P. Fibroblast Traction as a Mechanism for Collagen Morphogenesis. Nature. Vol. 290, No $5803249-251,1981$.

[23] M J Holmes, B D Sleeman. A Mathematical Model of Angiogenesis Incorporating Viscoelastic Effects $J$. Thoeretical Biology. Vol. 202, 95-112, 2000.

[24] James D W, Taylor J F. The Stress Developed by Sheets of Chick: Fibroblasts in Vitro. Exp. Cell Res. Vol. 54, 107-110, 1969.

[25] Landau L D, Lifshitz E M. Theory of Elasticity. Pergamon, London, 1959.

[26] Lackie J M. Cell Movement and Cell Behaviour. Allen and Unwin, London, 1986.

[27] Lauffenberger D A. A Simple Model for the Effects of Receptor Mediated Cell-Substratum Adhesion on Cell Migration. Chemical Engineering Science. Vol. 44(9), 1903$1914,1989$.

[28] Manoussaki D, Lubkin S R, Vernon R B, Murray J D. A Mechanical Model for the Formation of Vascular Networks in vitro. Acta Biotheoretica, Vol. 44, 271-282, 1996.

[29] Manoussaki D, Lubkin S R, Vernon R B, Murray J D. Simple Cellular Forces Form Vascular Networks in vitro. Acta Biotheoretica. 1997.

[30] Murray J D. Mathematical Biology. Springer-Verlag, New York, 1989.

[31] Murray J D, Oster G F. Cell Traction Models for the Generation of Pattern and Form in Morphogenesis. J. Math. Bio. Vol. $19,265-279,1984$
[32] Ngwa G A, Maini P K. Spatio-Temporal Patterns in a Mechanical Model for Mesenchymal Morphogenesis. Journal of Math. Biol. Vol. 33, 489-520, 1995.

[33] Odell G, Oster G, Burnside B, Alberch P. The Mechanical Basis of Morphogenesis, Epithelial Folding and Invagination. Dev Biology. Vol. 85, 446-462, 1981.

[34] Orme M E, Chaplain M A J. A Mathematical Model of the First Steps of Tumour-Related Angiogenesis: Capillary Sprout Formation and Secondary Branching. IMA Journal of Mathematics Applied in Medicine and Biology. Vol. 13, 73$98,1996$.

[35] Orme M E, Chaplain M A J. Two-Dimensional Models of Tumour Angiogenesis and Anti-Angiogenesis Strategies. IMA Journal of Mathematics Applied in Medicine and Biology. Vol. 14, 189-205, 1997.

[36] Oster G F, Perelson A S. Cell Spreading and Motility. A Model Lamellipod. J Math Biology. Vol. 21, 383-388, 1985.

[37] Oster G F, Perelson A S. The Physics of Cell Motility. J Cell Science Supplement 8, 33-54, 1987.

[38] Oster G F, Murray J D, Odell G M. The Formation of Microvilli. Molecular Determinants of Animal Form. New York: Alan R Liss. 365-384, 1985.

[39] Pettit E J, Fay F S. Cytosolic Free Calcium and the Cytoskeleton in the Control of Leukocyte Chemotaxis. Physiological Reviews. Vol. 78(4), 949-967, 1998.

[40] Skierczynski B A, Usami S, Skalak R. A Model of the Leukocyte Migration Through Solid Tissue Biomechanics of Active Movement and Division of Cells. Ed A Akkas NATO ASI series, Vol H 84.Springer-Verlag. 1994.

[41] Sleeman B D, Anderson A R A, Chaplain M A J. A Mathematical Analysis of a Model for Capillary Network Formation in the Absence of Endothelial Cell Proliferation. Appl. Math. Lett. Vol. 12, 121-127, 1999.

[42] Stokes C L, Lauffenberger D A, Williams S K. Migration of Individual Microvessel Endothelial Cells: Stochastic Model and Parameter Measurement. J. Cell. Sci. Vol. 99, 419-430, 1991.

[43] Trinkaus J P. Cells Into Organs. The Forces That Shape The Embryo Prentice-Hall, Inc, Englewood Cliffs, NJ. 1984.

[44] Yamada K M, Olden K. Fibronectins-Adhesive Glycoproteins of Cell Surface and Blood. Nature. Vol. 275, 179-184, 1978.

[45] Zaner K S, Vahlberg P A. Viscoelasticity of F-actin Measured with Magnetic Micro-particles. J Cell Biology. Vol. 109 2233-2243, 1989.

[46] Zhu C, Skalak R. A Continuum Model of Protrusion of Pseudopod in Leukocytes. Biophysical Journal. Vol. 54, 1115 $1137,1988$.

[47] Zienkiewicz O C. The Finite Element Method. McGraw-Hill London. 1977.

[48] Zigmond S. Chemotaxis by Polymorphonuclear Leukocytes. J. Cell Biology. Vol. 77, 269-287, 1978.

[49] Zigmond S, Sullivan S, Lauffenberger D. Kinetic Analysis of Chemotactic Peptide Receptor Modulation. J. Cell Biology. Vol. 92, 34-43, 1982 . 


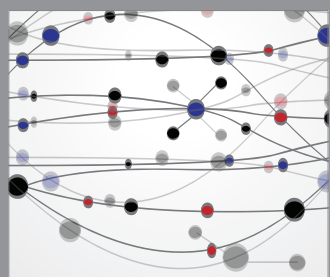

The Scientific World Journal
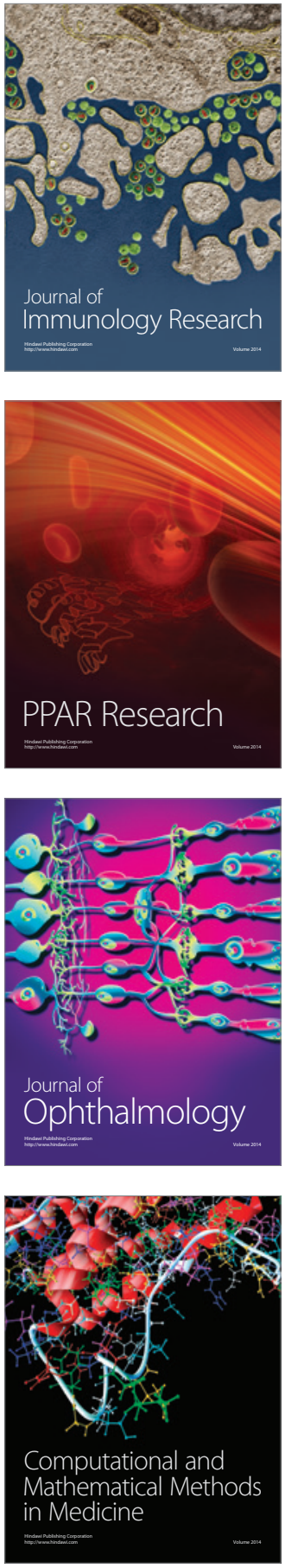

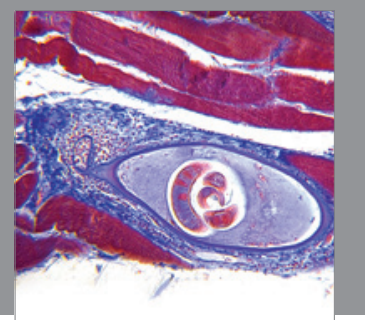

Gastroenterology

Research and Practice
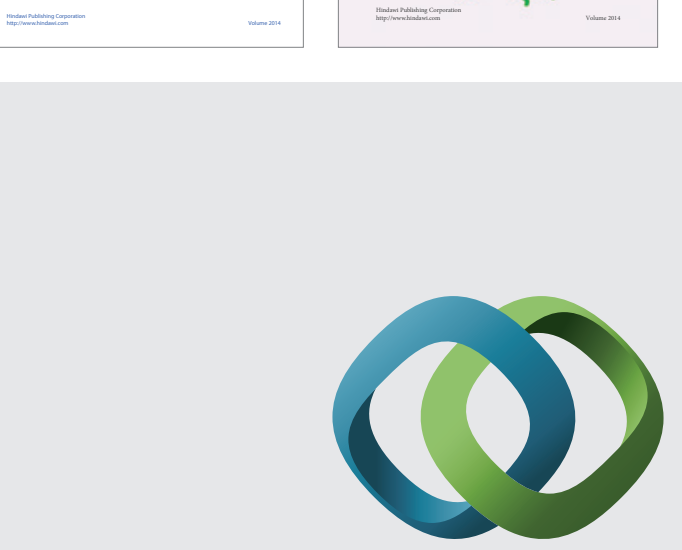

\section{Hindawi}

Submit your manuscripts at

http://www.hindawi.com
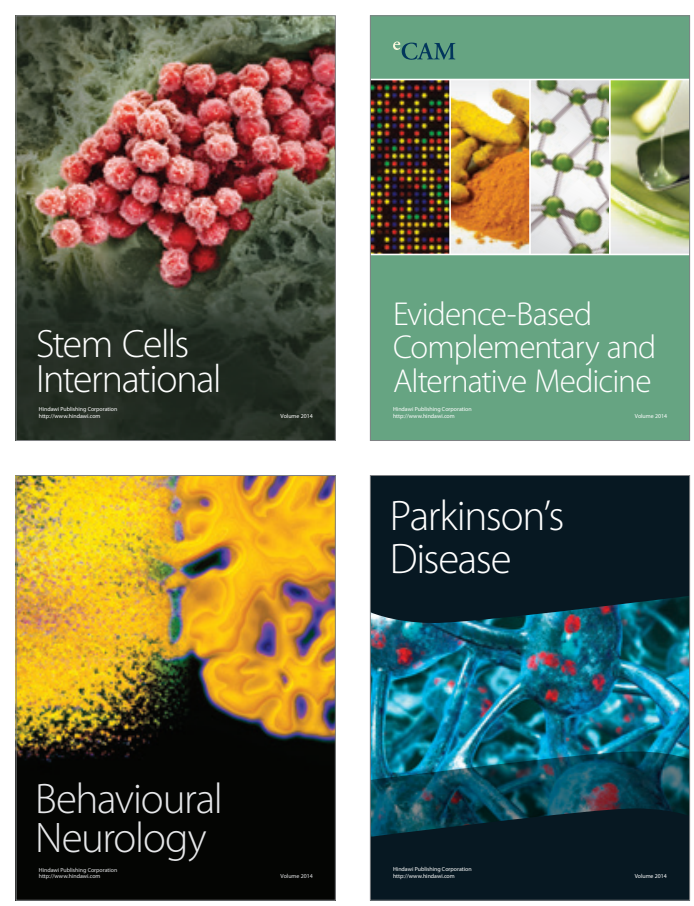

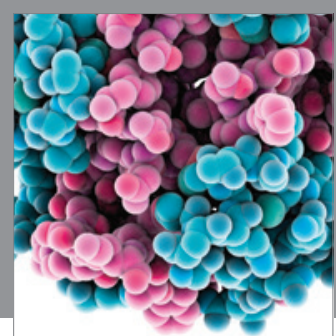

Journal of
Diabetes Research

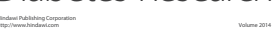

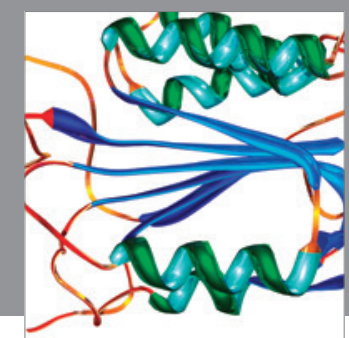

Disease Markers
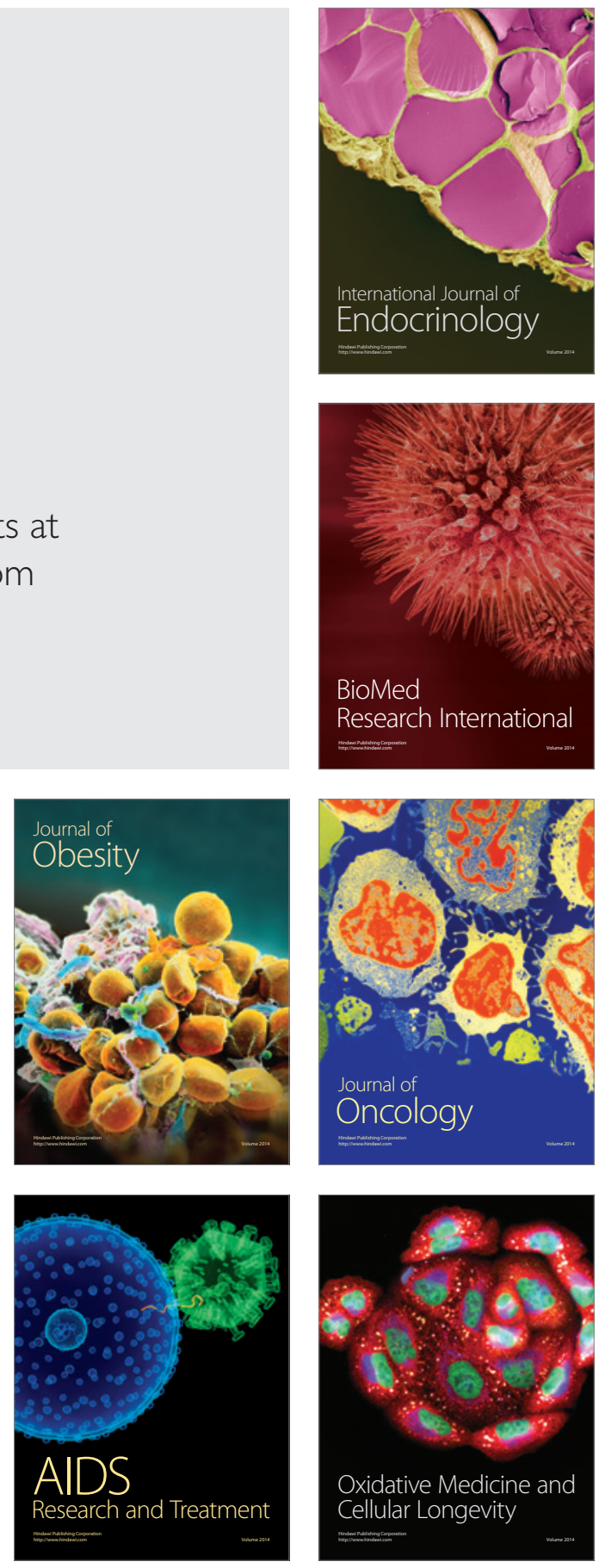